\title{
Schistosoma mansoni infection reprograms the metabolic potential of the myeloid lineage in
} a mouse model of metabolic syndrome

Diana Cortes-Selva ${ }^{1}$, Lisa Gibbs ${ }^{1}$, J. Alan Maschek ${ }^{5,6}$, Tyler Van Ry ${ }^{3,5}$, Bartek Rajwa ${ }^{2}$, James E.

$6 \mathrm{Cox}^{3,5}$, Eyal Amiel ${ }^{4}$, Keke C. Fairfax ${ }^{1,7}$

$8 \quad{ }^{1}$ Department of Pathology, Division of Microbiology and Immunology,

9 University of Utah, Salt Lake City UT, 84112

$10 \quad{ }^{2}$ Bindley Bioscience Center, Purdue University, West Lafayette IN, 47907

$11{ }^{3}$ Department of Biochemistry, University of Utah, Salt Lake City UT, 84112

$12{ }^{4}$ Department of Biomedical and Health Sciences, University of Vermont, Burlington, VT 05405

$13{ }^{5}$ Metabolomics, Proteomics and Mass Spectrometry Cores, University of Utah, Salt Lake City, UT, 1484112

$15{ }^{6}$ Department of Nutrition and Integrative Physiology and the Diabetes and Metabolism

16 Research Center, University of Utah, Salt Lake City, UT 84112

Running title: Schistosomes reprogram myeloid metabolism

${ }^{7}$ Lead contact:

23 Keke Fairfax

24 Department of Pathology

25 Division of Microbiology and Immunology

26 University of Utah

27 JMRB 2200B

$2815 \mathrm{~N}$ Medical Drive East

29 Salt Lake City, UT 84112

30 Email: keke.fairfax@path.utah.edu

31 ORCID: https://orcid.org/0000-0002-8382-2960

32 801-581-5980 (office)

Keywords: Myeloid lineage, macrophage metabolism, Schistosoma mansoni, biological sex, 


\section{Summary}

46 Despite evidence that helminths protect from metabolic disease, a major gap exists in

47 understanding the underlying mechanism(s). Here we demonstrate that bone marrow derived

48 macrophages (BMDM) from S. mansoni infected male ApoE ${ }^{-/-}$mice have dramatically increased

49 mitochondrial respiration compared to those from uninfected mice. This change associates with

50 increased glucose and palmitate shuttling into TCA cycle intermediates and decreased

51 accumulation of cellular cholesterol esters. Moreover, systemic metabolic modulation by

52 schistosomes is a function of biological sex, where infection protects $\mathrm{ApoE}^{-/-}$male, but not

53 female, mice from obesity and glucose intolerance. Sex-dependence extends to myeloid cells,

54 where reprogramming leads to opposite cholesterol phenotypes in BMDM from females and

55 males. Finally, the metabolic reprogramming of male myeloid cells is transferrable via bone

56 marrow transplantation to an uninfected host, indicating maintenance of reprogramming in the

57 absence of sustained antigen exposure. This work reveals that $S$. mansoni systemic reprograming

58 of myeloid metabolism is sex-dependent.

Introduction

62 Cardiovascular disease (CVD) is the leading worldwide cause of mortality (Hinton et al., 2018;

63 Roth et al., 2017). In the United States, $65 \%$ of adults diagnosed with diabetes have elevated

64 LDL cholesterol levels or take cholesterol lowering medications, and death rates from

65 atherosclerotic cardiovascular disease (CVD) are $\sim 1.7$ times higher in this population as

66 compared to non-diabetic adults (Emerging Risk Factors et al., 2010). It is well established that

67 in the diabetic population, obesity, and dyslipidemia are risk factors underlying these increases in 
mortality, while hyperglycemia is an independent risk factor (Marks and Raskin, 2000; Wong et al., 2016). Underlying conditions such as diabetes and atherosclerosis contribute to the burden of CVD in both females and males. While, the incidence of CVD is markedly higher in men than in age-matched women (Opotowsky et al., 2007; Tan et al., 2010), the risk of developing CVD while diabetic is much greater in women than men (Humphries et al., 2017; Peters et al., 2014). In non-diabetic patients, females exhibit increased insulin sensitivity in comparison to males, as well as reduced prevalence of dysglycemia and enhanced muscle glucose uptake (Cnop et al., 2003; Kim and Reaven, 2013; Moran et al., 2008; Willeit et al., 1997), suggesting sex-dependent modulations in whole body metabolism. Recent studies suggest a role of the gut microbiota in

77 the differences between sexes in the regulation of lipid metabolism (Baars et al., 2018).

78 Nevertheless, complete understanding of mechanistic basis behind sexual dimorphism in metabolic syndrome is still lacking.

81 Previous studies have uncovered an association between a history of helminth infection and 82 reduced prevalence of metabolic disease in humans and rodents ((Doenhoff et al., 2002; Stanley

83 et al., 2009; Wiria et al., 2015). Specifically, infection by Schistosomes reduces cholesterol and

84 atherosclerotic plaques (Doenhoff et al., 2002; Stanley et al., 2009), this effect has been

85 attributed, in part, to an anti-inflammatory phenotype in macrophages (Wolfs et al., 2014) and transcriptional reprogramming of phospholipid and glucose metabolism related genes in hepatic

87 macrophages (Cortes-Selva et al., 2018). Moreover, it has been postulated that schistosomes have the potential to affect long term glucose metabolism in T cells (Chen et al., 2013). 
91 studies have been conducted only in males or no sex differentiation has been made during data

92 analysis ((Sanya et al., 2019; Shen et al., 2015; Wolde et al., 2019)). To date, no report with a

93 particular emphasis on females has been conducted.

95 Schistosomiasis induces Th2 polarization and alternative activation of macrophages, essential for

96 host survival (Barron and Wynn, 2011; Fairfax et al., 2012; Herbert et al., 2004). IL-4 induced

97 alternative activation of macrophages relies on oxidative phosphorylation (OXPHOS) and fatty

98 acid oxidation for energy production, and is dependent on cell intrinsic lysosomal lipolysis

99 (Huang et al., 2014; Vats et al., 2006). Macrophage metabolism follows a dysmorphic pattern, as

100 sex-related differences affect the processes involved in cholesterol and lipid metabolism in

101 macrophages as well as inflammatory cytokine production in adipose tissue (Griffin et al., 2016;

$102 \mathrm{Ng}$ et al., 2001). Moreover, in rats, phagocytes from females had increased ROS generation than

103 males (Rudyk et al., 2018). Such differences have often been attributed to the role of sex

104 hormones in gene expression and immune cell function (Rubinow, 2018; Taneja, 2018; Winn et

105 al., 2019), but a clear understanding of the effects of sex on the regulation of macrophage

106 metabolism, as well as how sex modulates the effects of schistosomiasis in the protection from

107 metabolic disease is lacking.

109 In the present study, we sought to determine the systemic effects of $S$. mansoni infection on the

110 myeloid lineage. Surprisingly, we discovered that macrophages derived from the bone marrow of

111 S. mansoni infected male mice have dramatically increased oxygen consumption and

112 mitochondrial mass compared to those from uninfected males. This shift is accompanied by

113 increased carbon shuttling into TCA cycle intermediates, a decrease in cholesterol esters, and 
114 increased fatty acid oxidation. When we examined the role of biological sex in schistosome

115 induced modulation we found that S. mansoni infection does not reliably protect ApoE ${ }^{-/-}$female

116 mice from HFD induced weight gain or glucose intolerance. The sex-dependent effect of

117 infection extends to the myeloid lineage, where bone marrow derived macrophages from infected

118 females display the opposite metabolic phenotype as those from infected males, with a dramatic

119 increase in cellular cholesterol esters. Overall, these data present the first evidence that $S$.

120 mansoni systemically modulates the myeloid compartment in a sex-dependent manner and

121 provide a more comprehensive understanding of how S. mansoni infection may confer metabolic 122 protection at the cellular level.

\section{Results}

\section{Macrophages derived from $\boldsymbol{S}$. mansoni infected male mice have increased oxygen}

126 consumption and spare respiratory capacity

128 We have previously reported that schistosomiasis alters the expression of numerous genes

129 relevant to glucose, cholesterol, and amino acid metabolism in hepatic macrophages of male

130 mice (Cortes-Selva et al., 2018). These alterations are associated with improved insulin

131 sensitivity and atherosclerotic score in male mice. Since it has previously been shown that

132 during S. mansoni infection the majority of liver macrophages are monocyte derived, and

133 monocyte recruitment drives both atherosclerosis (Potteaux et al., 2011; Tacke et al., 2007) and

134 obesity induced insulin resistance (Beliard et al., 2017; Liang et al., 2007; Oh et al., 2012; Rull et

135 al., 2010), we hypothesized that Schistosome infection may imprint the monocyte- macrophage

136 lineage with altered metabolic propensities. To elucidate whether infection imprints 
137 macrophages with an altered metabolic phenotype we infected (and mock infected controls)

138 atherogenesis-prone male ApoE ${ }^{-/}$mice on high-fat diet (HFD), and sacrificed them at 10-weeks

139 post-infection to harvest bone marrow cells. Macrophages were differentiated in vitro with M-

140 CSF in a 6-7-day culture. We performed real-time extracellular flux analysis on unstimulated

141 bone marrow derived macrophages (BMDM) from $\mathrm{ApoE}^{-/-} \mathrm{HFD}$ infected and uninfected

142 (control) HFD mice to quantify oxygen consumption rate (OCR) (Figure 1A). BMDM from

$143 \mathrm{ApoE}^{-/-} \mathrm{HFD}$ infected mice showed improved basal respiration (Figure 1B) and significantly

144 increased spare respiratory capacity $(\mathrm{p}<0.0001$, Figure $1 C)$. Since eukaryotic cells integrate

145 oxidative phosphorylation (OXPHOS), glycolysis and the tricarboxylic acid (TCA) cycle to

146 satisfy energy requirements, we also tested the extracellular acidification rate (ECAR), which is

147 suggested as a marker of inhibited mitochondrial respiration (Pike Winer and $\mathrm{Wu}, 2014$ ), in

148 BMDM from infected and uninfected ApoE ${ }^{-/-}$HFD mice. We observed no differences in ECAR

149 in infected male mice compared to uninfected controls (Figure 1D). Cell intrinsic lysosomal

150 lipolysis has previously been shown to support macrophage spare respiratory capacity in the

151 context of macrophage alternative activation (Huang et al., 2014; Liu et al., 2017). We stained

152 for hydrophobic and neutral lipids by Oil Red O (ORO) (Mehlem et al., 2013) and observed that

153 the lipid content of BMDM from infected $\mathrm{ApoE}^{-/-}$males trended to reduction in comparison to

154 the control group, but was not significantly reduced (Figure 1E). To analyze BMDM

155 mitochondrial mass, which has also been linked to increased respiratory capacity (Langston et

156 al., 2017), we analyzed mitochondrial activity by Mitotracker Deep Red FM. We observed that

157 BMDM from infected mice exhibited increased MitoTracker median fluorescent intensity (MFI)

158 in comparison to the BMDM from uninfected mice (Figure 1F). Overall, these data indicate that

159 S. mansoni infection in males leads to increased oxygen consumption and mitochondrial 
160 metabolism in BMDM. Mitochondrial oxidative dysfunction in macrophages has recently been

161 linked to insulin resistance (Jung et al., 2018), so this metabolic shift could contribute to the

162 infection-induced improvement in glucose tolerance seen in infected males .

164 Schistosomiasis in male $\mathrm{ApoE}^{-/-}$mice alters metabolic flux of glucose and the lipidomic

165 fingerprint of macrophages

167 In order to understand how Schistosome infection alters the metabolic fingerprint and promotes 168 mitochondrial metabolism in macrophages derived from $\mathrm{ApoE}^{-/-} \mathrm{HFD}$ male mice, we performed

169 metabolic tracing analysis, where macrophages were differentiated in the presence of normal

170 glucose and then switched to ${ }^{13} \mathrm{C}$-labeled glucose for 24 hours. We observed increased shuttling

171 of heavy labeled glucose to malate (Figure 2A), citrate (Figure 2B), itaconate (Figure 2C), and

172 succinate Figure 2E in BMDM from infected mice in comparison to BMDM from uninfected

173 mice. The lack of increased heavy lactate production (Figure 2D), suggests that the primary

174 reprogramming is focused on glucose-dependent mitochondrial metabolism. Importantly, we

175 found no upregulation of alternative activation markers (CD301, CD206, Arg1, Nos2) in

176 unstimulated BMDM from infected mice when compared to BMDM from controls

177 (Supplementary Figure 1). Since we have previously shown that infection alters the phospholipid

178 and cholesterol metabolism in hepatic macrophages (Cortes-Selva et al., 2018), we performed

179 unbiased lipidomics using liquid chromatography-mass spectrometry (LC-MS). We used a

180 supervised model by partial least squares-discriminant analysis (PLS-DA), with two components

181 to determine the lipidomic profile of BMDM. There was a robust separation between groups that

182 indicates the metabolic profiles of BMDM from infected and uninfected males differ 
183 significantly and suggests that there is a prominent alteration of metabolites induced by infection

184 in male mice (Figure 2F). The lipid species that drive the variation observed in the PLS-DA as

185 measured by the Variable Importance in Projection (VIP) score from uninfected males in

186 comparison to infected males included cholesterol esters (CE) (20:1), CE (22:0), CE (24:0), CE

187 (24:1), CE (22:4), CE (22:1), CE (18:0); diacylglycerols (DG) (16:0_16:0), DG (16:0_18:0) and

188 triacyclglycerol (TG) (16:0_16:0_16:0) (Figure 2G,H). We then analyzed the total CE levels in

189 macrophages from both groups of male mice and found that infection led to significantly reduced

190 CE in male mice $(\mathrm{p}<0.0001$, Figure 2I), further evidencing an important role of cholesterol

191 metabolism in macrophages following helminth infection. Next, we determined the

192 transcriptomic modifications induced by infection in unstimulated BMDM from infected and

193 uninfected males by mRNA sequencing (mRNAseq). Significant gene expression differences

194 were observed in BMDM from infected male mice, compared to uninfected controls. Transcripts

195 from the two groups are depicted in Volcano plots, using false discovery rate (FDR $<0.05$ in red,

196 FDR $<0.01$ in blue) and $\log 2$ fold changes (cut off of .6 Log2 FC) to identify statistically

197 significant genes (Figure 2J). Among the differentially regulated factors are Gbp6, which is

198 related to interferon- $\gamma$ signaling and innate immune function; Gm7609, a predicted pseudogene;

199 Gbp4, a member of the GTPase family involved in protective immunity against microbial and

200 viral pathogens; Apo19a, which is predicted to be related to lipid transport, lipoprotein metabolic

201 processes and stimulated by interferon; Iigp1, a GTPase with roles in response to intracellular

202 pathogens; CD300e, which belongs to families of paired activating and inhibitory receptors

203 implicated in immune responses; and Batf2, a basic leucine zipper factor whose activation is

204 detrimental for type-1 infectious disease. Importantly, Mgll, which encodes monoacylglycerol

205 lipase that catalyzes the conversion of monoacylglycerides to free fatty acids and glycerol was 
significantly increased. Mgll is required for lipolysis and improved glucose homeostasis in mice on HFD (Berdan et al., 2016; Douglass et al., 2015), an infection driven increase was validated by RT-qPCR (Figure 2L). In addition, Slc1a3, which encodes for the glutamate aspartate transporter 1 that is localized in the inner mitochondria membrane as part of the malate-aspartate

210 shuttle and is relevant for amino acid homeostasis in adipocytes, was significantly altered in our

211 model, and was further validated by RT-qPCR (Figure 2M). Interestingly, the pathways

212 significantly altered (Figure $2 \mathrm{~K})$ during infection included hematopoietic cell lineage $(p=5.779$

$\left.213 \times 10^{-8}\right)$, asthma $\left(\mathrm{p}=3.169 \times 10^{-5}\right)$, and cytokine-cytokine receptor interactions, graft-versus host

214 disease, type 1 diabetes and allograft regression $(\mathrm{p}=0.0000894)$. Together, these data identify key

215 factors in immune and metabolic responses as well as novel factors with unknown functions that 216 are regulated by exposure to $S$. mansoni infection.

S. mansoni infection protects male mice, but not female mice, from obesity and glucose intolerance independently of systemic alternative macrophage activation

221 Sex is a key contributor to the phenotype of cardiovascular and metabolic features in mammals

222 (Chella Krishnan et al., 2018), so we assessed the sex dependent impact of S. mansoni infection 223 on obesity and glucose intolerance. For this, we fed male and female ApoE ${ }^{-/}$HFD for 10-days

224 before infection. We infected and mock infected mice (controls) as described in the methods.

225 Ten weeks post infection we analyzed body weight and glucose tolerance (via an IP glucose 226 tolerance test) and found that infection is significantly beneficial for male, but not female mice, 227 as only males are protected from HFD-induced weight gain and glucose intolerance (Figure 3A, 228 3B). We also analyzed serum triglyceride (TG) and diglyceride (DG) levels using untargeted 
229 lipidomics and found that relative abundance of both TG and DG were decreased in infected

230 males as compared to uninfected males, while TGs were increased by infection in females and

231 DGs were unchanged (Figure 3C). These data indicate that S. mansoni infection induces a sex-

232 dependent modulation of metabolic disease parameters. We then wondered if this infection-

233 mediated effect in males only was correlated with differences in systemic alternative activation

234 of hepatic macrophages in females. Flow cytometry analysis showed that alternative activation

235 markers (CD206, CD301, Arg1) were highly expressed in macrophages from male and female

236 mice following infection, but not in naïve mice (Figure 3D), suggesting that $S$. mansoni induced

237 alternative activation irrespective of sex. Previous studies characterizing the dynamics of

238 alternatively activated macrophages during schistosome infection have found that these

239 macrophages primarily arise from Ly6C ${ }^{\text {high }}$ monocytes (Girgis et al., 2014; Nascimento et al.,

240 2014). Naïve male and female $\mathrm{ApoE}^{-/-}$mice on HFD had equivalent frequencies of Ly6chigh

241 monocytes circulating in peripheral blood. At 10-weeks post infection we found an increased

242 percentage of both Ly6C $\mathrm{C}^{\text {int }}$ and Ly6C $\mathrm{C}^{\text {high }}$ cells (Figure 3E) in both male and female mice

243 compared to the mock infected controls, with the frequency of Ly6C $\mathrm{C}^{\text {high }}$ cells in females 1.74

244 times that of males, suggesting either increased monopoiesis in females, or increased tissue

245 recruitment in males. Since we had found increased mitochondrial MFI in BMDM from infected

246 male mice, we asked whether circulating blood monocytes are similarly modulated. We observed

247 an infection induced increase in Mitotracker fluorescent intensity in monocytes from male ApoE-

248 /- mice on HFD, but not from females (Figure 3F). These data suggest that there is sex-specific

249 increased mitochondrial activity following infection in the monocyte cell population. We then

250 wondered if these effects in the differentiated monocytes are the result of long-lasting changes in

251 the myeloid lineage after helminth infection. For this, we analyzed the main lineages of 
252 hematopoietic progenitors that produce myeloid cells: granulocyte-monocyte progenitors (GMP),

253 monocyte-DC progenitors (MDP) and the common myeloid progenitor (CMP) in female and

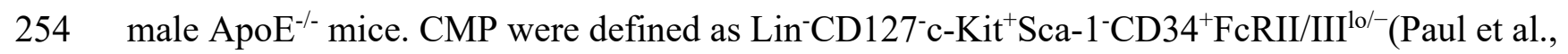

255 2016), GMP were defined as $\mathrm{Lin}^{-} \mathrm{IL}-7 \mathrm{R}^{-} \mathrm{Sca}-1^{-} \mathrm{c}-\mathrm{kit}^{+} \mathrm{CD} 34^{+} \mathrm{FcR} \mathrm{II} / \mathrm{III}{ }^{+}$, and MDP were defined

256 as defined as $\mathrm{Lin}^{-} \mathrm{c}-\mathrm{Kit}^{+} \mathrm{Sca}-1^{-} \mathrm{CD} 34^{+} \mathrm{Fc} \gamma \mathrm{R}^{\mathrm{lo}} \mathrm{CD} 115^{\mathrm{hi}}$ cells. Overall bone marrow cellularity

257 was not affected by Schistosome infection (Figure 3G), however, the numbers of CMP and GMP

258 in infected male mice were significantly reduced compared to uninfected controls, while GMP

259 and CMP were not reduced in females (Figure $3 \mathrm{H}-\mathrm{J}$ ). The numbers and percentages of MDP

260 remained unchanged in infected females and males compared to uninfected controls (Figure 3K,

261 3L). These data suggest that S. mansoni infection modulates both metabolic disease and the

262 myeloid lineage in a sex-specific manner that is independent from the induction of systemic

263 alternative activation.

S. mansoni-increases fatty acid oxidation in male but not female ApoE ${ }^{-/-}$mice on HFD

267 In order to determine whether infection-induced pathologic differences in males and females

268 were accompanied by sex-specific differential macrophage metabolic regulation, we cultured

269 bone marrow cells from 10-week infected or uninfected control male and female mice for 7 days

270 to generate BMDM. OCR was measured in real time in basal conditions and following the

271 addition of mitochondrial inhibitors in unstimulated BMDM from both infected and uninfected

272 male and female ApoE ${ }^{-/-}$animals. Similar to as seen in Figure 1A, BMDM from infected males

273 exhibited increased basal OCR and spare respiratory capacity compared to uninfected male

274 controls (Figure 4A, 4B, 4C). However, basal OCR and spare respiratory capacity of BMDM 
275 from infected females remained unaltered in comparison to BMDM from uninfected females

276 (Figure 4A, 4B, 4C). Moreover, side by side OCR analysis in females and males with palmitate

277 as a substrate (glucose limiting conditions) showed that BMDM from infected male, but not from

278 infected females had an increased ability to oxidize exogenous palmitate (Figure 4D). In

279 addition, BMDM from infected males but not females had significantly increased palmitate basal

280 OCR and palmitate spare respiratory capacity, suggesting exogenous free fatty usage as a carbon

281 source for OXPHOS (Figure 4E, 4F). Similar to the male only data, we observed no differences

282 in macrophage lipid content in either group, suggesting that global lipolysis may not underlie

283 OCR and spare respiratory capacity in our model (Figure 4G). Additionally, analysis of

284 mitochondrial mass in females and males showed that similar to blood monocytes, BMDM from

285 infected male mice, but not females have a significantly higher Mitotracker MFI. These data

286 suggest that $S$. mansoni infection improved mitochondrial biogenesis in males, but not females.

287 Again, suggesting differential regulation of macrophage metabolism based on biological sex.

288 This regulation may account in part for the differences in the role of infection in modulating

289 obesity and insulin sensitivity.

S. mansoni infection differentially alters the cellular lipid profile in female and male mice

293 To understand the sex specific modulations induced by schistosomiasis in macrophage lipid

294 metabolism, we isolated cellular lipids from unstimulated BMDM and performed unbiased

295 lipidomics in male and female derived-cells. PLS-DA showed that S. mansoni infection led to a

296 sex-specific lipid profile (Figure 5A). Further, analysis of total CE (identified as a VIP species in

297 males in Figure 2) showed that infection led to decreased levels of CE in cells derived from 
298 infected male mice. In contrast, infection led to significantly increased total CE in BMDM

299 derived from infected female mice (Figure 5B). Similar to males, infection in females induces a

300 unique lipid signature (Figure 5C), with two species of plasmanyl-PE, three species of

301 plasmanyl-PC, BMP and CE as the main drivers of the altered lipid profile in females during

302 infection (Figure 5D). Since we observed BMP's as a VIP compound driven by S. mansoni

303 infection in females, but not males, we went back and performed targeted lipidomics to quantify

304 BMPs at the class level. Indeed, total BMPs were significantly increased by infection in BMDM

305 from females but not males (Figure 5E). Our Seahorse data indicates that S. mansoni infection

306 induced differential oxidation of exogenous palmitate in BMDM derived from males and

307 females. In order to understand the metabolic flux of exogenous palmitate, we performed

308 metabolic tracing analysis of palmitate, where macrophages were differentiated in the presence

309 of normal glucose and then switched to $\mathrm{C}^{13}$-labeled palmitate for 24 hours. Similar to what we

310 documented with glucose, we observed increased shuttling of heavy labeled palmitate into

311 succinate, malate, and fumarate in BMDM derived from infected males, with decreased shuttling

312 into myristate (a marker of fatty acid elongation). BMDM derived from infected females

313 displayed the opposite phenotype, with decreased shuttling of heavy labeled palmitate into

314 succinate, malate, and fumarate, and increased shuttling into myristate compared to BMDM from

315 uninfected controls. These data suggest that BMDM derived from infected male $\mathrm{ApoE}^{-/-}$mice

316 have increased basal utilization of exogenous palmitate for beta-oxidation, while the female data

317 suggests increased lipid synthesis, strengthening the data obtained from our palmitate

318 extracellular flux analysis.

319 To further understand the role of biological sex in the S. mansoni induced reprograming of the

320 BMDM lipidome, we isolated cellular lipids from male and female BMDM either unstimulated 
321 (media), or following LPS stimulation, and then performed untargeted lipidomics as described in

322 Figure 5. We analyzed the lipidomic data with machine learning using a two-step selection

323 process (see Methods). This approach identified 20 features (lipid compounds) contributing to

324 an elastic net regression model that can predict the infection status of samples-originating

325 animals (Zou, 2005). These informative features are listed in descending order of importance

326 (Supplemental Figure 2A). Interestingly, some key features (for instance, the top scoring one) are

327 not represented as significant in the univariate tests (univariate adjusted $\mathrm{p}$ values, Supplemental

328 Figure 2B). However, these features are important contributors to the multivariate machine

329 learning model. This is not unexpected - some features that would be considered uninformative

330 individually may be very useful in improving predictions if combined with other features. When

331 we examine these features individually, we can see a differential response to infection for males

332 and females (for instance, PG 18:1_18:2, a glycerophospholipid) represented in Supplemental

333 Figure 2C). Seven of the 20 molecular features identified by machine learning are BMP

334 (Bis(monoacylglycerol)phosphate) species, which have been implicated in glycosphingolipid

335 degradation and cholesterol transport (Anheuser et al., 2019; Luquain-Costaz et al., 2013). These

336 data further support the hypothesis that S. mansoni infection differentially modulates the

337 metabolism of myeloid cells from male and female animals, and that this modulation revolves

338 around decreased cholesterol storage and fatty acid synthesis in males and increased cholesterol

339 storage and fatty acid synthesis in females. Cholesterol and lipid metabolism has previously been

340 associated with inflammatory myeloid effector function (Carroll et al., 2018; Funk et al., 1993;

341 Oiknine and Aviram, 1992), so we quantified the acute inflammatory effectors nitrite and iNOS,

342 along with pro-inflammatory cytokines/chemokines IL-12p70, CXCL1, and IL-6

343 (chemokines/cytokines with known pathogenic roles in obesity, insulin resistance and 
atherosclerosis) following stimulation with LPS. S. mansoni infection increases LPS induced nitrite and iNOS production in BMDM from male, but not female ApoE ${ }^{-/-}$mice on HFD (Figure and IL-6 (Figure 5 L-N) following LPS stimulation as compared to BMDM from uninfected controls. LPS induced IL-12p70, CXCL1, and IL-6 production by female BMDM is unaffected by infection status. Increased production of the effector molecules nitrite and iNOS combined with decreased production of pro-inflammatory mediators associated with chronic inflammation supports the idea that $S$. mansoni infection promotes a hybrid macrophage state in males.

\section{S. mansoni infection modulates the myeloid transcriptome in a sex-specific manner}

354 Since we documented significant sex dependent shifts in functional metabolism in BMDM from modulation underlies these shifts. In order to investigate the genes and respective pathways that

363 metabolism we identified hexokinase 1 (hk1), citrate synthase (cs), apolipoprotein A2 (apoa2),

364 aldehyde dehydrogenase 3 family member A2 (aldh3a2), lipoyltransferase (lipt1), solute carrier

365 family 19 member 1 (slc19a1), LDL receptor related protein 1 (lrp1), many of these are involved 366 in lipoprotein and cholesterol metabolism. These data suggest that myeloid metabolism is 
367 differentially regulated by sex, at least in the context of HFD. In addition, we found 216 genes

368 involved in immunity. Among these genes with immune function we identified interleukin 10 (il-

369 10), Toll like receptor 5 (tlr5), NLR family pyrin domain containing 3 (nlrp3), inducible T cell

370 costimulatory (icos), which have diverse pro and anti-inflammatory function in the immune

371 system. Next, we surveyed the genes that are differentially regulated in males and females

372 following S. mansoni infection. We found 66 genes involved in metabolism, hemostasis, the

373 adaptive immune system, collagen degradation and not annotated to specific pathways.

374 Following the genes with known function, the majority (10) of differentially regulated genes

375 have documented roles in metabolism. Among these, we identified type II iodothyronine

376 deiodinase (dio2), which has been implicated in the regulation of diet induced obesity

377 (Kurylowicz et al., 2015; Vernia et al., 2013). Moreover, we found that hexokinase 3 (hk3), fatty

378 acid binding protein 4 (fabp4), sphingomyelin synthase 2 (sgms2), solute carrier family 6

379 member 8 (slc6a8) were all upregulated in male and downregulated in female BMDM following

380 S. mansoni infection (Figure 6B). In addition, we performed gene ontology analysis from the 66

381 genes that were differentially regulated in females and males. The most significant pathways

382 were response to glucocorticoids, glycoprotein metabolism, and fatty acid metabolism, again

383 suggesting that there is a sex-specific metabolic response to $S$. mansoni infection in myeloid

384 cells. These pathways may help explain the differential metabolic modulation induced by

385 schistosomiasis in males and females (Figure 6C).

386

387 S. mansoni induced modulation of male macrophage metabolism is long-lived in the 
Our metabolic and transcriptomic data from BMDM differentiated in vitro from male $S$. mansoni infected mice suggested that metabolic modulation may be long-lived in the absence of ongoing

391 antigen exposure. In order to determine durable nature of modulation we transferred bone marrow from either 10 -week S. mansoni infected male ApoE ${ }^{-/-}$mice on HFD, or uninfected male controls into busulfan treated recipient $\mathrm{ApoE}^{-/-}$mice on HFD. At 10 weeks post-bone marrow

394 transfer we assayed glucose tolerance via an i.p. GTT. Mice that received bone marrow from 395 infected males have a significantly lower glucose area under the curve (AUC) than those that

396 received control bone marrow. We harvested bone marrow from all recipients and differentiated

397 BMDM in M-CSF for 6 days and then performed real-time extracellular flux analysis. BMDM

398 from recipients of bone marrow from S. mansoni infected mice had significantly higher basal 399 oxygen consumption and a trend towards increased spare respiratory capacity as compared to

400 BMDM generated from recipients of uninfected control bone marrow. Additionally, BMDM

401 from recipients of bone marrow from S. mansoni infected mice had a significantly higher

402 Mitotracker MFI than BMDM from recipients of control bone marrow. These data suggest that $S$.

403 mansoni induced metabolic modulation of the myeloid lineage in males is long-lived even in the

404 absence of ongoing exposure to egg antigens, and that hematopoietic cells are at least partially

405 responsible for the regulation of whole-body glucose metabolism in the HFD ApoE ${ }^{-/-}$model.

\section{Discussion}

Helminth infections in general, and schistosomiasis in specific, have been known to be

408 inversely correlated with obesity and glucose intolerance for over a decade, a phenomenon

409 thought to be associated with Type 2 polarization of macrophages and T cells. In the current

410 study we report that $S$. mansoni infection induces dramatic metabolic alterations in BMDM from

411 male $\mathrm{ApoE}^{-/-}$mice on HFD. Our results indicate that macrophages derived from the bone marrow 
412 of infected male mice have increased basal oxygen consumption and spare respiratory capacity

413 compared to those derived from uninfected males. In T cells, an increase in spare respiratory

414 capacity has been linked to mitochondrial biogenesis, and controls the transition to a long-lived

415 memory phenotype (van der Windt et al., 2012). In macrophages, M2 (alternative) activation has

416 previously been shown to lead to increased spare respiratory capacity, a process that also

417 involves mitochondrial biogenesis (Kannan et al., 2016), while TLR recognition of bacteria has

418 been shown to increase mitochondrial respiration via modulation of complex I and II (Garaude et

419 al., 2016). In both cell types, the increased mitochondrial respiration underlies the longevity of

420 the cells. In our model we have not found most of the traditional markers used to define M2

421 alternative activation by flow cytometry at steady state (Supplemental Figure 1). Arg1 is the only

422 canonical M2 transcript that is modulated in the BMDM from infected male mice at steady state,

423 and that fold increase is relatively low (.894 log FC, adjusted $p$-value $=.035)$. Arg 1 drives the

424 production of polyamines, which in turn are able to modulate mitochondrial OxPHOS (Galvan-

425 Pena and O'Neill, 2014; Puleston et al., 2019). In the currently accepted paradigm of M2

426 polarization stat6 phosphorylation upregulates PGC1- $\beta$ and PPAR $\gamma$, leading to mitochondrial

427 biogenesis and increased beta-oxidation in addition to Arg1transcription. Here we present a

428 model where neither PGC1- $\beta$ or PPAR $\gamma$ are modulated, but Arg1 transcription is increased with

429 a concomitant dramatic increase in mitochondrial respiration, suggesting that there may be

430 alternative ways to modulate mitochondrial metabolism.

431 Alternative activation has previously been shown to be dependent on cell intrinsic

432 lysosomal lipolysis and lal, with the defining feature being reductions in lipid droplets (Huang et

433 al., 2014). In our model using unstimulated BMDM from S. mansoni infected mice there is no

434 gross difference in lipid droplets, nor an increase in lal transcripts. Instead, we found a 
435 significant shift in the lipidome of BMDM from infected male mice that centered on a reduction

436 in cholesterol esters. Previously published work in IL-4 induced M2 macrophages has found that

437 lipolysis centered on TGs as a fatty acid source. While we did find reductions in two species of

438 DGs and one species of monoacylglycerol (MG), there were no significant alterations to TAGs

439 in the BMDM generated from infected males. Flux analysis with heavy carbon labeled glucose

440 and palmitate suggest that BMDM from male infected mice have increased shuttling of both

441 glucose and palmitate into TCA cycle intermediates, suggesting that these cells have increased

442 mitochondrial beta oxidation. In addition to the unique lipidomic modulations, we found that the

443 dramatic increase in both basal oxygen consumption and spare respiratory capacity we observed

444 in BMDM from male infected mice was significantly palmitate dependent. This observation

445 further supports the idea that in vivo exposure of myeloid precursors to helminth antigens

446 induces unique metabolic modulations that focus on cholesterol and lipid metabolism as a source

447 for palmitate for beta oxidation. These data suggest that hybrid metabolic states in the absence of

448 M1 or M2 polarization occur in macrophages in the context of chronic disease, and present a

449 challenge to the dichotomy of M1 versus M2 activation being driven by immunometabolism.

450 Future studies exploring the immunometabolism of bone marrow derived and tissue resident

451 macrophages and dendritic cells from other chronic infections and inflammatory diseases are

452 needed in order to obtain a true picture of the correlation between metabolism and myeloid

453 polarization.

454 There are significant clinical differences in both the etiology and pathology of diabetes

455 and cardiovascular disease between males and females, but sex differences in immunological

456 activation or modulation by $S$. mansoni infection have not previously been studied in humans or

457 animal models. Surprisingly, we found that the unique metabolic modulations induced by $S$. 
mansoni infection in BMDM from male mice do not occur in females, and infected females are not reliably protected from high-fat diet induced obesity or glucose intolerance. Interestingly,

460 hepatic macrophage alternative activation in response to infection is equivalent between males

461 and females, but infection increases blood monocyte frequency in females to a much greater

462 extent than in males, suggesting sex specific modulation of either monopoiesis or monocyte

463 recruitment into tissues. Blood monocytes from infected males phenocopy the increase in

464 Mitotracker MFI that we have found in BMDM generated from infected males, indicating that

465 our BMDM model is likely an accurate representation of the in vivo potential of the myeloid 466 compartment. Interestingly, we found that infection significantly reduced the total number of

467 CMP and GMP in male, but not female bone marrow, again pointing to a sex-specific

468 modulation of the myeloid lineage at the precursor level. The reduction of CMP and GMP in

469 infected males suggests either an increase in the rate of differentiation into

470 monocytes/granulocytes, or decreased homeostatic proliferation. Since males have fewer blood

471 monocytes than female, we favor the later possibility. These possibilities and the relationship

472 between reductions in numbers of CMP and GMP in males, and the functional metabolic

473 differences in BMDM will be explored in future work.

Analysis of the BMDM transcriptomes from males and females revealed that over a

476 thousand genes are up regulated in male versus female BMDM regardless of S. mansoni

477 infection state. At the same time, over five dozen are differentially regulated by infection.

478 Focusing on the genes that are up-regulated in BMDM from infected males and down-regulated

479 in females, we found more than ten genes with known functions in cellular metabolism. Some of 480 these genes, like PFKFB3, have known regulatory elements for progesterone and estrogen (Shi et 
481 al., 2017), but some of them, like fabp4, have no published mechanism of sex hormone

482 regulation. Glycolysis and cholesterol metabolism have previously been shown to directly affect

483 the inflammatory potential of myeloid cells. Our data indicate that $S$. mansoni infection induces a 484 hybrid inflammatory state in male but not female BMDM, where the LPS induced production of

485 nitrite and iNOS is enhanced, while the production of the key chronic pro-inflammatory

486 mediators IL-12p70, CXCL1, and IL-6 are reduced. This inflammatory profile is distinct from

487 what has previously been published with IL-4 induced M2 macrophages, where the production of 488 iNOS and nitrite are reduced following TLR stimulation (Lam et al., 2016). CXCL1 and IL-6 489 have previously been linked to increased monocyte recruitment and disease progression in both 490 atherosclerosis and obesity-induced diabetes (Boisvert et al., 2006; Hartman and Frishman, 2014;

491 Nunemaker et al., 2014; Qu et al., 2014; Zhou et al., 2011), so these data support the possibility

492 of infection driven modulation of macrophage function supporting the decreased pathology seen

493 in infected males. We have demonstrated that the modulation of macrophage oxygen

494 consumption is transferrable to an uninfected recipient, and can last for at least ten weeks,

495 suggesting that in males, $S$. mansoni infection induces long-lived metabolic modulation of the

496 myeloid lineage that survives in the absence of ongoing antigenic exposure. Trained innate

497 immunity has previously been documented to be induced by BCG immunization (Kaufmann et

498 al., 2018), and has recently been suggested to be the mechanism underlying the association

499 between previous bacterial and fungal infections and the development of atherosclerosis

500 (Hoogeveen et al., 2018; Leentjens et al., 2018). In these models, trained immunity and

501 epigenetic reprogramming is driven in part from a switch from oxidative phosphorylation to

502 increased aerobic glycolysis (Cheng et al., 2014; Stienstra et al., 2017). In the case of BCG, 503 trained circulating monocytes can be found months after immunization, which strongly suggests 
504 reprogramming of bone marrow progenitors (Ifrim et al., 2014). Recent reports indicate that

505 western high-fat diet itself also induces innate training of bone marrow progenitors in both the

$506 \mathrm{Ldr}^{-/-}$model of atherosclerosis (Christ et al., 2018) and in obesity related steatohepatitis (Krenkel

507 et al., 2020). Our data suggests that $S$. mansoni infection in males trains the myeloid lineage in

508 the opposite fashion; modulating the metabolic transcriptome of the myeloid lineage such that

509 oxidative phosphorylation and mitochondrial activity is increased, while the chronic

510 inflammatory potential is decreased. Alterations to the numbers and frequency of myeloid

511 progenitors and the transferability of our phenotype via bone marrow suggests that progenitors

512 are indeed modulated in our model, the relative role of epigenetic modulation versus microRNA

513 regulation in dictating the $S$. mansoni induced myeloid transcriptome will be the subject of future

514 studies.

515 There are significant differences in both the susceptibility and disease presentation

516 between males and females for both diabetes and cardiovascular disease, but few studies have

517 focused on the role of immunometabolism in this dichotomy. Our data suggests apparent

518 biological sex-dependent differences in both the ability of schistosomes to protect from the

519 development of HFD induced metabolic disease parameters, and the ability to modulate

520 macrophage glucose and lipid metabolism. The current epidemiological data strongly indicates

521 an inverse correlation between helminth infections and metabolic diseases such as diabetes and

522 cardiovascular disease, but these studies were not designed to identify sex-specific correlations.

523 Few animal studies have focused on the role of myeloid cells in driving sex-specific metabolic

524 differences. Our current data indicate a clear need for further studies in both humans and animal

525 models to specifically probe the relationship between biological sex and myeloid metabolism,

526 and how chronic helminth infections modulate this. 


\section{Conflict of Interest}

529 The authors declare no competing interests.

530 Acknowledgements

531 The work was supported by The University of Utah, a Scientist Development Grant from the

532 American Heart Association to KCF (14SDG18230012), an American Heart Association Pre-

533 doctoral Award (18PRE34030086) to DCS, and 1R21AI135385-01A1 to EA. B. glabrata snails

534 provided by the NIAID Schistosomiasis Resource Center of the Biomedical Research Institute

535 (Rockville, MD) through NIH-NIAID Contract HHSN272201700014I for distribution through

536 BEI Resources. JEC is supported through U54 DK110858-01, mass spectrometry equipment

537 employed was provided by 1S10OD016232-01, 1S10OD018210-01A1 and 1S10OD021505-01

538 to JEC.

STAR Methods

\section{KEY RESOURCES TABLE}

\begin{tabular}{|l|l|l|}
\hline REAGENT or RESOURCE & SOURCE & IDENTIFIER \\
\hline Antibodies & \multicolumn{2}{l|}{} \\
\hline Anti-mouse F4/80 (Clone: BM8) & Biolegend & Cat no. 123114 \\
\hline $\begin{array}{l}\text { Anti-mouse CD64 a and b Alloantigens (Clone: X54- } \\
\text { 5/7.1.1) }\end{array}$ & BD Biosciences & Cat no. 558455 \\
\hline Biotinylated Goat Anti-m-Mer & R\&D Systems & Cat no. BAF591 \\
\hline Anti-mouse CD11c (Clone: N418) & BioLegend & Cat no. 117306 \\
\hline Rat Anti-mouse I-A/I-E (Clone: M5/114.15.2) & BD Biosciences & Cat no. 562366 \\
\hline Anti-mouse Ly6A/E (Sca-1) (Clone: D7) & eBiosciences & Cat no.17-5981-82 \\
\hline Rat anti-mouse CD117/c-kit & R\&D Systems & Cat no. FAB1356 \\
\hline Anti-mouse CD115 (Clone: AFS98) & eBiosciences & Cat no. 25-1152-82 \\
\hline Anti-mouse CD16/32 (Clone: 93) & Invitrogen & Cat no.25-0161-82 \\
\hline Anti-mouse CD4 (Clone: GK1.5) & BioLegend & Cat no.100406 \\
\hline Anti-mouse CD206 (Clone:C06C2) & BioLegend & Cat no. 141721 \\
\hline Rat Anti-mouse CD301 & BioRad & $\begin{array}{l}\text { Cat no. } \\
\text { MCA2392A488 }\end{array}$ \\
\hline Anti-mouse Nos2 (Clone: CXNFT) & Cat no. 46-5920-80 \\
\hline
\end{tabular}




\begin{tabular}{|c|c|c|}
\hline Anti $\mathrm{Hu} / \mathrm{Mo}$ Arg1 (Clone: A1exF5) & Invitrogen & Cat no. $48-3697-80$ \\
\hline Anti-mouse CD19 (Clone: MB19-1) & Invitrogen & Cat no.11-0191-85 \\
\hline Anti-mouse Ter119 (Clone: Ter-119) & Invitrogen & Cat no. 11-5921082 \\
\hline Rat Anti-mouse Ly6G and Ly6C (Clone: RB6-8C5) & BD Biosciences & Cat no. 553127 \\
\hline Anti-mouse CD3 (Clone: 17A2) & BioLegend & Cat no. 100204 \\
\hline Anti-mouse Ly6C (Clone: HK1.4) & Invitrogen & Cat no. $45-5932-82$ \\
\hline Anti-mouse CD45 (Clone 30-F11) & Biolegend & Cat no. 103154 \\
\hline Anti-mouse CD11b (Clone: M1/70) & eBiosciences & Cat no. $56-0112-82$ \\
\hline MitoTracker ${ }^{\mathrm{TM}}$ Deep Red FM & Invitrogen & Cat no. M22426 \\
\hline Anti-mouse CD34 (Clone: SA376A4) & BioLegend & Cat no. 152203 \\
\hline TrueStain fcX (anti-mouse CD16/32) (Clone: 93) & BioLegend & Cat no. 101320 \\
\hline Anti-mouse CD127 (Clone: A7R34) & BioLegend & Cat no. 135043 \\
\hline \multicolumn{3}{|l|}{ Biological Samples } \\
\hline Fetal Bovine Serum & ThermoFisher & Cat no. 10091148 \\
\hline \multicolumn{3}{|l|}{ Chemicals, Peptides, and Recombinant Proteins } \\
\hline Endotoxin free LPS (Escherichia coli Serotype O) & InvivoGen & Cat no. tlrl-eblps \\
\hline D-Glucose- ${ }^{13} \mathrm{C} 6$ & Sigma Aldrich & Cat no. 389374 \\
\hline Recombinant murine M-CSF & Peprotech & Cat no. 315-03 \\
\hline BD Pharm Lyse Lysing Buffer & BD Biosciences & Cat no. 555899 \\
\hline Taqman Gene Expression Master Mix & Applied Biosystems & Cat no. 4369016 \\
\hline Trypan Blue solution & Sigma & T81154-100ML \\
\hline Streptavidin APC & BD Biosciences & Cat no. 554067 \\
\hline Streptavidin APC/Fire 750 & Biolegend & Cat no. 405250 \\
\hline Streptavidin PE & BD Biosciences & Cat no. 554061 \\
\hline Streptavidin PB & Invitrogen & Cat no. S11222 \\
\hline Streptavidin PE-Cy5 & BD Biosciences & Cat no. 554062 \\
\hline (Ethylenedinitrilo)tetraacetic acid (EDTA) & $\begin{array}{l}\text { Thermo Fisher } \\
\text { Scientific }\end{array}$ & Cat no. AM9260G \\
\hline \multicolumn{3}{|l|}{ Fixation/Permeabilization } \\
\hline Collagenase & Sigma Aldrich & Cat no. 5138 \\
\hline Trizol LS Reagent & Ambion & Cat no. 10296028 \\
\hline Chloroform & Alfa Aesar & Cat no. 32614 \\
\hline Glucose & Sigma Aldrich & Cat no. G7528 \\
\hline 2-Propanol & Fisher Chemical & Cat no. A416 \\
\hline \multicolumn{3}{|l|}{ Critical Commercial Assays } \\
\hline Seahorse Bioassay & Agilent Technologies & Cat no. 102416 \\
\hline SuperScript IV-VILO Master Mix & Thermo Fisher & Cat no. 11766050 \\
\hline RNA Clean and Concentrator Kit & Zymo Research & Cat no. 11-353 \\
\hline Zombie Red Fixable Viability Kit & BioLegend & Cat no.423109 \\
\hline${ }^{13} \mathrm{C}$ Glucose & Santa Cruz Biotech & $\begin{array}{l}\text { Cat no. 106032-62- } \\
6\end{array}$ \\
\hline${ }^{13} \mathrm{C}$ Palmitate & Sigma-Aldrich & Cat no. 605573 \\
\hline Cellstripper cell dissociation reagent & Corning & Cat no. 25-056-CI \\
\hline
\end{tabular}


bioRxiv preprint doi: https://doi.org/10.1101/2020.04.20.050898; this version posted May 21, 2020. The copyright holder for this preprint (which was not certified by peer review) is the author/funder, who has granted bioRxiv a license to display the preprint in perpetuity. It is made available under aCC-BY 4.0 International license.

\begin{tabular}{|c|c|c|}
\hline Western High-fat diet & Envigo & TD 88137 \\
\hline \multicolumn{3}{|l|}{ Deposited Data } \\
\hline GEO & $\begin{array}{l}\text { https://www.ncbi.nlm. } \\
\text { nih.gov/geo/ }\end{array}$ & GSE144447 \\
\hline \multicolumn{3}{|l|}{ Experimental Models: Organisms/Strains } \\
\hline B6.129P2-Apoetm1Unc/J & $\begin{array}{l}\text { The Jackson } \\
\text { Laboratory }\end{array}$ & Stock No: 002052 \\
\hline Parasite: Schistosoma mansoni & $\begin{array}{l}\text { Biomedical Research } \\
\text { Institute }\end{array}$ & $\begin{array}{l}\text { https://www.afbr- } \\
\text { bri.org/schistosomia } \\
\text { sis/ordering/ }\end{array}$ \\
\hline \multicolumn{3}{|l|}{ Software and Algorithms } \\
\hline FlowJo Software X 10.0.7r2 & BD Biosciences & $\begin{array}{l}\text { https://www.flowjo.c } \\
\text { om/solutions/flowjo/ } \\
\text { downloads/previous- } \\
\text { versions }\end{array}$ \\
\hline GraphPad Prism 8.2.0 & GraphPad & $\begin{array}{l}\text { https://www.graphpa } \\
\text { d.com/support/faq/pr } \\
\text { ism-820-release- } \\
\text { notes/ }\end{array}$ \\
\hline Attune NxT Accoustic Focusing Cytometer & Invitrogen & $\begin{array}{l}\text { https://www.thermof } \\
\text { isher.com/us/en/hom } \\
\text { e/life-science/cell- } \\
\text { analysis/flow- } \\
\text { cytometry/flow- } \\
\text { cytometers/attune- } \\
\text { acoustic-focusing- } \\
\text { flow-cytometer.html }\end{array}$ \\
\hline iPathways Guide & Advaita & $\begin{array}{l}\text { https://advaitabio.co } \\
\text { m/ipathwayguide/ }\end{array}$ \\
\hline MH Qual & $\begin{array}{l}\text { Agilent Technologies, } \\
\text { Inc }\end{array}$ & $\begin{array}{l}\text { https:/www.agilent. } \\
\text { com/en/products/soft } \\
\text { ware- } \\
\text { informatics/masshun } \\
\text { ter- } \\
\text { suite/masshunter/ma } \\
\text { sshunter-software }\end{array}$ \\
\hline MassHunter Quantitative Analysis B.07.00 & $\begin{array}{l}\text { Agilent Technologies, } \\
\text { Inc }\end{array}$ & $\begin{array}{l}\text { https://www.agilent. } \\
\text { com/en/products/soft } \\
\text { ware- } \\
\text { informatics/masshun } \\
\text { ter- } \\
\text { suite/masshunter/ma } \\
\text { sshunter-software }\end{array}$ \\
\hline LipidMatch & University of Florida & $\begin{array}{l}\text { http://secim.ufl.edu/s } \\
\text { ecim- } \\
\text { tools/lipidmatch/ }\end{array}$ \\
\hline MetaboAnalyst 3.0 & Xia et al., 2015 & $\begin{array}{l}\text { http://www.metaboa } \\
\text { nalyst.ca }\end{array}$ \\
\hline Other & & \\
\hline
\end{tabular}




\begin{tabular}{|l|l|l|}
\hline Mgll Taqman Gene Expression Assay & ThermoFisher & $\begin{array}{l}\text { Assay ID: } \\
\text { Mm00449274_m1 }\end{array}$ \\
\hline Slc1a3 Taqman Gene Expression Assay & ThermoFisher & $\begin{array}{l}\text { Assay ID: } \\
\text { Mm00600697_m1. }\end{array}$ \\
\hline Beta actin Taqman Gene Expression Assay & ThermoFisher & $\begin{array}{l}\text { Assay ID: } \\
\text { Mm00607939_s1 }\end{array}$ \\
\hline
\end{tabular}

\section{LEAD CONTACT AND MATERIALS AVAILABILITY}

544 Further information requests should be directed to and will be fulfilled by the Lead Contact,

545 Keke Fairfax (keke.fairfax@path.utah.edu). These studies generated no new reagents.

\section{EXPERIMENTAL MODEL AND SUBJECT DETAILS}

$548 \quad$ Parasite and Mouse Models

549 This study was carried out in accordance with the recommendations in the Guide for the Care

550 and Use of Laboratory Animals of the National Institutes of Health. The protocols were

551 approved by the Institutional Animal Care and Use Committees of the University of Utah and

552 Purdue University. Snails infected with S. mansoni (strain NMRI, NR-21962) were provided by

553 the Schistosome Research Reagent Resource Center for distribution by BEI Resources, NIAID

554 NIH. Male and female ApoE-/- (B6.129P2-Apoetm1Unc/J) were purchased from the Jackson

555 Laboratories and bred at the University of Utah. Mice were housed in pathogen-free conditions

556 and were fed standard rodent chow (2019 rodent chow, Harlan Teklad) until 10-14 days before

557 infection when they were transitioned to a high-fat diet (HFD: $21 \%$ milk fat, $0.15 \%$ cholesterol:

558 TD 88137 Envigo). Bone marrow chimeras were generated by treating ApoE ${ }^{-/-}$mice that had

559 been on high- fat diet for 4 weeks with $20 \mathrm{mg} / \mathrm{kg}$ of pharmaceutical grade busulfan for 5 days

560 (total dose of $100 \mathrm{mg} / \mathrm{kg}$ ). On day 6 mice were i.v. injected with $2.5-3 \times 10^{6}$ bone marrow cells

561 from either 10-week S. mansoni infected or control uninfected ApoE ${ }^{-/-}$mice on high-fat diet. 
562 Reconstitution was validated via flow-cytometry at 3-weeks post-transfer and recipient mice

563 were maintained on high-fat diet for 10-weeks post-reconstitution.

564 METHOD DETAILS

565 S. mansoni infection and glucose tolerance test

$566 \mathrm{ApoE}^{-/-}$female and male mice of 6 weeks of age were exposed percutaneously to 75-90 cercariae

567 of $S$. mansoni or were mocked infected (as controls). At five- and ten-weeks post-infection mice

568 were fasted for 5 hours and baseline blood glucose levels were obtained via lateral tail vein nick.

569 Mice were then administered a single intraperitoneal injection of glucose $(2 \mathrm{mg} / \mathrm{g}$ of body weight,

570 ultrapure glucose, Sigma G7528). Blood glucose levels were obtained at 20, 60, and 90 min post

571 injection. Individual data points obtained were analyzed by Area Under Curve (AUC).

572

573 Mouse macrophage culture

574 Mouse bone marrow-derived macrophages (BMDM) were generated as follows: bone marrow

575 cells were isolated by centrifugation of bones at $>10,000 \mathrm{xg}$ in a microcentrifuge tube for 15

576 seconds as previously described (Amend et al., 2016). Cells were differentiated in M-CSF

577 (20ng/mL, Peprotech, Rocky Hill, NJ) in complete macrophage medium (CMM: RPMI1640,

$57810 \%$ FCS, $2 \mathrm{mM}$ L-glutamine and $1 \mathrm{IU} / \mathrm{mL}$ Pen-Strep for 6 or 7 days. On the last day, cells

579 were harvested in Cellstripper cell dissociation reagent (Corning) were washed with CMM) and

580 prepared for downstream assays.

Glycolytic and phospho-oxidative metabolism measurement (seahorse assay)

583 BMDM from different conditions (uninfected controls or $S$. mansoni infected) were resuspended 584 at the same concentration in XF assay media supplemented with 5\% FCS and 5mM glucose. The 
585 day before the assay, the probe plate was calibrated and incubated at $37 \mathrm{C}$ in a non-CO2

incubator. Resuspended cells were seeded at a concentration of $1.5 \times 10^{5}$ cells per well and

587 incubated for 20-60 minutes in the Prep Station incubator (37 C non-CO2 incubator). Following

588 initial incubation, XF Running Media (XF assay media with 5\% FCS and 10mM Glucose) were

589 dispensed into each well. OCR and ECAR were measured by an XF96 Seahorse Extracellular

590 Flux Analyzer following the manufacturer's instructions. For the seahorse assay, cells were

591 treated with oligomycin $(1 \mathrm{uM}), \mathrm{FCCP}(1.5 \mathrm{uM})$, rotenone $(100 \mathrm{nM})$ and antimycin A $(1 \mathrm{uM})$. Each

592 condition was performed in 2-3 technical replicates. For determination of palmitate dependent

593 respiration, $\mathrm{BSA}$-conjugated palmitate $(\mathrm{BSA}$ : palmitate $=1: 6$, molar ratio) was prepared

594 according to the Seahorse protocol (Seahorse Bioscience). Briefly, $1 \mathrm{mM}$ sodium palmitate

595 (Sigma Aldrich) was conjugated with $0.17 \mathrm{mM}$ fatty acid free-BSA (Sigma Aldrich) in $150 \mathrm{mM}$

$596 \mathrm{NaCl}$ solution at $37^{\circ} \mathrm{C}$ for $1 \mathrm{~h}$. Palmitate-BSA was stored in glass vials at $-20^{\circ} \mathrm{C}$ until use. Cells

597 were incubated as above in glucose limited XF media per manufacturer instructions.

599 Flow Cytometry

600 Livers from uninfected and infected mice were perfused with 1X PBS, mashed and digested in 601 DMEM $0.5 \%$ Collagenase (Sigma) at $37^{\circ} \mathrm{C}$ for $15 \mathrm{~min}$. Then, livers were mashed and filtered 602 through a $100 \mu \mathrm{m}$ metal strainer and digested an additional $15 \mathrm{~min}$. Following second digestion, 603 the liver contents were strained, washed with DMEM and spun down $1500 \mathrm{rpm}$ for $5 \mathrm{~min}$. The 604 pellet was lysed with 1X lysis buffer (BD PharmLyse), quenched with 1\% FBS DMEM, and 605 washed to be used in flow cytometry. Surface staining was performed using the following mAb 606 against mouse antigens: CD45 (30-F11, eBioscience), CD301(BioRad), CD206 (C068C2, 607 Biolegend), F4/80 (BM8, Biolegend), mouse Mer biotinylated (R\&D), CD64(X54-5/7.1, BD). 
608 Intracellular antigen staining such as Nos2 (CXNFT, Invitrogen, and C-11, Santa Cruz) and Arg1

609 (Invitrogen, A1exF5)) was performed using the Intracellular Fixation and Permeabilization

610 Buffer set (Thermo Fisher Scientific cat. no. 88-8824) per manufacturer's instruction. Further,

611 bone marrow cells were obtained by centrifugation of bones into tubes at $>10000 \mathrm{rpm}$ for $15 \mathrm{~s}$.

612 Surface staining for bone marrow precursors was performed using the following antibodies:

613 Ter119, CD19, CD4, CD3, Gr-1, CD11b, Sca-1, CD115, Ly6C, c-Kit, flt3, CD127 and CD16/32.

614 PBMC from whole blood were obtained following red blood cell lysis and used for flow

615 cytometry analysis. Surface staining of PBMC was performed using Ter119, CD64, CD11b,

616 CD115, Ly6C and MitoTracker Red.

617 Samples were acquired using Attune NxT Focusing Flow Cytometer (Thermo Fisher Scientific)

618 and analyzed using Flowjo X 10.0.7r2 (FlowJo LLC, Inc.).

620 RNA Isolation and q-RT-PCR

621 BMDM were stored in Trizol, and RNA isolation was performed as described in the

622 Immunological Genome Project Total RNA isolation protocol. Next, cDNA was synthesized

623 from RNA using Superscript IV VILO (ThermoFisher Scientific) for reverse transcription. qPCR

624 was performed using TaqMan Gene expression assays (Mgll, Slc1a3, beta actin, ThermoFisher)

625 on an Applied Biosystems Stepone Plus Real-Time PCR System. Beta-Actin assay number

626 Mm00607939_s1, mgll assay Mm00449274_m1, slc1a3 assay Mm00600697_m1. Relative

627 expression was calculated using the $2-\Delta \Delta \mathrm{Ct}$ method.

628

629 Measurement of Cytokines and Inflammatory mediators 
630 For cytokine levels of BMDCs, supernatants were collected at 24 hours post stimulation and

631 measured with Mouse Cytokine and Chemokine ProcartaPlex 26plex panel (Life Technologies)

632 per manufacture instructions using a Luminex Magpix system. Nitrite levels in cell culture media

633 were determined using a Griess reagent kit for nitrite determination (Invitrogen) according the

634 manufacturer's instructions.

\section{Untargeted lipidomics}

637 Sample extraction from serum or cell pellets

638 Lipids were extracted from serum $(50 \mu \mathrm{L})$ or cell pellets in a combined solution as described in

639 (Matyash et al., 2008). In detail, samples were combined in solution with $225 \mu \mathrm{L} \mathrm{MeOH}$

640 containing internal standards (IS; Avanti splash Lipidomix (Lot\#12), 10 $\mu \mathrm{L}$ each sample) and 750

$641 \mu \mathrm{L}$ methyl tert-butyl ether (MTBE). The samples were sonicated for $1 \mathrm{~min}$, rested on ice for 1

642 hour, briefly vortexed every $15 \mathrm{~min}$ then an addition of $200 \mu \mathrm{L}$ dd-H2O was made to induce

643 phase separation. All solutions were pre-chilled on ice. The sample were then vortexed for $20 \mathrm{~s}$,

644 rested at room temperature for $10 \mathrm{~min}$, and centrifuged at $14,000 \mathrm{~g}$ for $10 \mathrm{~min}$ at $4 \mathrm{C}$. The upper

645 organic phase was collected and evaporated to dryness under vacuum. Lipid samples were

646 reconstituted in $200 \mu \mathrm{L}$ IPA and transferred to an LC-MS vial with insert for analysis.

647 Concurrently, a process blank sample was brought forward as well as quality control sample was

648 prepared by taking equal volumes $(10 \mu \mathrm{L}$ per sample) from each sample after final resuspension.

\section{LC-MS Methods}

651 Lipid extracts were separated on a Waters Acquity UPLC CSH C18 1.7 um 2.1 x 100 mm 652 column maintained at $65^{\circ} \mathrm{C}$ connected to an Agilent HiP 1290 Sampler, Agilent 1290 Infinity 
653 pump, equipped with an Agilent 1290 Flex Cube and Agilent 6530 Accurate Mass Q-TOF dual

654 ESI mass spectrometer. For positive mode, the source gas temperature was set to $225^{\circ} \mathrm{C}$, with a

655 gas flow of $11 \mathrm{~L} / \mathrm{min}$ and a nebulizer pressure of 50 psig. VCap voltage was set at $3500 \mathrm{~V}$,

656 fragmentor at $110 \mathrm{~V}$, skimmer at $85 \mathrm{~V}$ and Octopole RF peak at $750 \mathrm{~V}$. For negative mode, the

657 source gas temperature was set at $325^{\circ} \mathrm{C}$, with a drying gas flow of $12 \mathrm{~L} / \mathrm{min}$ and a nebulizer

658 pressure of 30 psig. VCap voltage is set $3000 \mathrm{~V}$, fragmentor at $125 \mathrm{~V}$, skimmer at $75 \mathrm{~V}$ and

659 Octopole RF peak at $750 \mathrm{~V}$. Reference masses in positive mode $(\mathrm{m} / \mathrm{z} 121.0509$ and 922.0098$)$

660 were infused with nebulizer pressure at $2 \mathrm{psig}$, in negative mode reference masses $(\mathrm{m} / \mathrm{z}$

$661 \quad 1033.988,966.0007,112.9856$ and 68.9958) were infused with a nebulizer pressure at 5psig.

662 Samples were analyzed in a randomized order in both positive and negative ionization modes in

663 separate experiments acquiring with the scan range $\mathrm{m} / \mathrm{z}$ 100-1700. Mobile phase A consisted of

$664 \mathrm{ACN}: \mathrm{H}_{2} \mathrm{O}(60: 40 v / v)$ in $10 \mathrm{mM}$ ammonium formate and $0.1 \%$ formic acid, and mobile phase B

665 consisted of IPA:ACN: $\mathrm{H}_{2} 0(90: 9: 1 v / v)$ in $10 \mathrm{mM}$ ammonium formate and $0.1 \%$ formic acid.

666 The chromatography gradient for both positive and negative modes started at $15 \%$ mobile phase

667 B then increased to $30 \%$ B over $2.4 \mathrm{~min}$, then increased to $48 \%$ from $2.4-3.0$ min, followed by an

668 increase to $82 \%$ B from 3-13.2 min, and then to $99 \%$ from 13.2-13.8 min where it was held until

$66915.4 \mathrm{~min}$ and then returned to the initial conditioned and equilibrated for $4 \mathrm{~min}$. Flow was 0.5

$670 \mathrm{~mL} / \mathrm{min}$ throughout, injection volume was $5 \mu \mathrm{L}$ for positive and $7 \mu \mathrm{L}$ negative mode. Tandem

671 mass spectrometry is conducted using the same LC gradient at collision energies of $20 \mathrm{~V}$ and 40

$672 \mathrm{~V}$.

673

674 Targeted lipidomics

675 Sample Preparation 
676 Lipids were extracted from cell pellets (500,000 cells) as described in detail above (Matyash et

677 al., 2008). For targeted lipidomics, lipid extracts were separated on a Waters BEH HILIC column

$6781.7 \mu \mathrm{m}, 100 \mathrm{~mm} \times 3 \mathrm{~mm}$ column maintained at $60{ }^{\circ} \mathrm{C}$ connected to an Agilent HiP 1290

679 Sampler, Agilent 1290 Infinity pump, and equipped with an Agilent 6490 triple quadrupole

680 (QqQ) mass spectrometer. Lipids were detected using dynamic multiple reaction monitoring

681 (dMRM) in negative ion mode. Source gas temperature is set to $225^{\circ} \mathrm{C}$, with a gas flow of 13

$682 \mathrm{~L} / \mathrm{min}$ and a nebulizer pressure of $30 \mathrm{psi}$. Sheath gas temperature was $350{ }^{\circ} \mathrm{C}$, sheath gas flow

683 was $11 \mathrm{~L} / \mathrm{min}$, capillary voltage of $4000 \mathrm{~V}$, nozzle voltage was $500 \mathrm{~V}$, high pressure RF was 190

$684 \mathrm{~V}$ and low-pressure RF was $120 \mathrm{~V}$. Injection volume was $2 \mu \mathrm{L}$ and the samples were injected in

685 a randomized order. Mobile phase A consisted of $\mathrm{H}_{2} \mathrm{O}$, mobile phase $\mathrm{B}$ consisted of $\mathrm{ACN}: \mathrm{H}_{2} \mathrm{O}$

$686(96: 4 \mathrm{v} / \mathrm{v})$ and both contain $7 \mathrm{mM}$ ammonium acetate. The chromatography gradient started at

$687100 \%$ mobile phase B and decreased to $84 \%$ B over 10 min. Post-time was 9 min and the flow

688 rate was $0.4 \mathrm{~mL} / \mathrm{min}$ throughout. Collision energies $(25 \mathrm{~V})$ and cell accelerator voltages $(3 \mathrm{~V})$

689 were optimized using lipid standards with dMRM quantifier transitions as $[\mathrm{M}-\mathrm{H}]^{-} \rightarrow\left[\mathrm{T} 2{ }_{-} \mathrm{RCOO}^{-}\right.$

690 and qualifier transitions of $[\mathrm{M}-\mathrm{H}]^{-} \rightarrow\left[\mathrm{T}_{-} \mathrm{RCOO}^{-}\right.$and $[\mathrm{M}-\mathrm{H}]^{-} \rightarrow\left[\mathrm{NL} \_\mathrm{T} 2 \mathrm{Ketene}\right]^{-}$.

\section{Lipid data analysis}

693 The pooled QC samples and process blank samples were injected throughout the sample queue to

694 ensure the reliability of acquired LC-MS data. Results from LC-MS experiments were collected

695 using Agilent MassHunter (MH) Workstation and analyzed using the software packages $\mathrm{MH}$

696 Qual, MH Quant (Agilent Technologies, Inc) and LipidMatch to prepare the data set.

697 The data table exported from MHQuant was evaluated using Excel where initial lipid targets

698 were parsed based on the following criteria. Only lipids with relative standard deviation (RSD) 
699 less than 30\% in QC samples were used for data analysis. Additionally, targets identified in

700 blanks or double blanks at significant amounts (area under the curve (AUC) target blank/AUC

701 target QC $>30 \%$ ) were removed from analysis. Lipids were quantitated based on peak area ratios

702 to the spiked IS of the same or nearest class.

\section{RNA Sequencing}

705 Library Preparation and sequencing

706 The concentration and quality of total RNA samples was first assessed using Agilent 2100

707 Bioanalyzer. A RIN (RNA Integrity Number) of five or higher was required to pass the quality

708 control. Then 200 nanograms of RNA per sample were used to prepared dual-indexed strand-

709 specific cDNA library using KAPA mRNA Hyperprep Kit (Roche). The resulting libraries were

710 assessed for its quantity and size distribution using Qubit and Agilent 2100 Bioanalyzer. Two

711 hundred pico molar pooled libraries were utilized per flowcell for clustering amplification on

712 cBot using HiSeq 3000/4000 PE Cluster Kit and sequenced with 2.75bp paired-end configuration

713 on HiSeq4000 (Illumina) using HiSeq 3000/4000 PE SBS Kit. A Phred quality score (Q score)

714 was used to measure the quality of sequencing. More than $90 \%$ of the sequencing reads reached

715 Q30 (99.9\% base call accuracy).

717 Sequence alignment and gene counts

718 The sequencing data were first assessed using FastQC (Babraham Bioinformatics, Cambridge,

719 UK) for quality control. Then all sequenced libraries were mapped to the mouse genome (UCSC

720 mm10) using STAR RNA-seq aligner (Dobin et al., 2013) with the following parameter: "--

721 outSAMmapqUnique 60". The reads distribution across the genome was assessed using bamutils 
722 (from ngsutils) (Breese and Liu, 2013). Uniquely mapped sequencing reads were assigned to

723 mm10 refGene genes using featureCounts (from subread) (Liao et al., 2014) with the following

724 parameters: “-s $2-\mathrm{p}-\mathrm{Q}$ 10”. Quality control of sequencing and mapping results was summarized

725 using MultiQC (Ewels et al., 2016). Genes with read count per million $(C P M)>0.5$ in more than

7262 of the samples were kept. The data was normalized using TMM (trimmed mean of M values)

727 method. Differential expression analysis was performed using edgeR (McCarthy et al., 2012;

728 Robinson et al., 2010). False discovery rate (FDR) was computed from p-values using the

729 Benjamini-Hochberg procedure.

730 Pathway analysis

731 The Data (significantly impacted pathways, biological processes, molecular interactions.) were

732 analyzed using Advaita Bio's iPathwayGuide (http://www.advaitabio.com/ipathwayguide).

733 Pathway analysis was performed on $\log _{2}$-transformed data using Bonferroni-corrected $p$-values.

734 The data discussed in this publication have been deposited in NCBI's Gene Expression Omnibus

735 (Edgar et al., 2002) and are accessible through GEO Series accession number GSE

736 144447(https://www.ncbi.nlm.nih.gov/geo/query/acc.cgi?acc=GSE144447)

737

738 Heavy glucose and heavy palmitate labeling

739 For metabolomics tracing in Figure 2 BMDM were differentiated in CMM containing normal

740 glucose. At Day 6 of culture cells were switched to $\mathrm{CMM}$ containing ${ }^{13} \mathrm{C}_{6}$-glucose (Santa Cruz

741 Biotech) for 24 hours. Cells were harvested and processed as described below. For metabolomics

742 tracing in figure $5 \mathrm{BMDM}$ were differentiated in CMM containing normal glucose and serum. At

743 Day 6 of culture cells were switched to CMM containing dialyzed serum and $1 \mathrm{mM}^{13} \mathrm{C}$

744 palmitate (sigma Aldrich) for 36 hours. Cells were harvested and processed as described below. 


\section{Metabolomics}

\section{Extraction}

749 Cold $90 \%$ methanol $(\mathrm{MeOH})$ solution was added to each sample to give a final concentration of

$75080 \% \mathrm{MeOH}$ to each cell pellet. Samples were incubated at $-20{ }^{\circ} \mathrm{C}$ for $1 \mathrm{hr}$. After incubation, the

751 samples were centrifuged at $20,000 \times$ g for 10 minutes at $4{ }^{\circ} \mathrm{C}$. The supernatant was transferred

752 from each sample tube into a labeled, fresh micro centrifuge tube. The samples were dried en

753 vасио.

Mass Spectrometry Analysis of Samples

756 All GC-MS analysis was performed with an Agilent 7200 GC-QTOF and an Agilent 7693A

757 automatic liquid sampler. Dried samples were suspended in $40 \mu \mathrm{L}$ of a $40 \mathrm{mg} / \mathrm{mL} \mathrm{O}-$

758 methoxylamine hydrochloride (MOX) (MP Bio \#155405) in dry pyridine (EMD Millipore

$759 \quad$ \#PX2012-7) and incubated for one hour at $37{ }^{\circ} \mathrm{C}$ in a sand bath. $25 \mu \mathrm{L}$ of this solution was

760 added to auto sampler vials followed by the automatic addition of $60 \mu \mathrm{L}$ of N-methyl-N-

761 trimethylsilyltrifluoracetamide (MSTFA with 1\%TMCS, Thermo \#TS48913) and incubated for

76230 minutes at $37^{\circ} \mathrm{C}$. Following incubation, each sample were vortexed and $1 \mu \mathrm{L}$ of the prepared

763 sample was injected into the gas chromatograph inlet in the split mode with the inlet temperature

764 held at $250^{\circ} \mathrm{C}$. A 10:1 split ratio was used for analysis for the majority of metabolites. Any

765 metabolites that saturated the instrument at the 10:1 split was analyzed at a 50:1 split ratio. The

766 gas chromatograph had an initial temperature of $60^{\circ} \mathrm{C}$ for one minute followed by a $10^{\circ} \mathrm{C} / \mathrm{min}$

767 ramp to $325^{\circ} \mathrm{C}$ and a hold time of 10 minutes. A 30-meter Agilent Zorbax DB-5MS with $10 \mathrm{~m}$ 
Duraguard capillary column was employed for chromatographic separation. Helium was used as the carrier gas at a rate of $1 \mathrm{~mL} / \mathrm{min}$.

\section{Data Analysis}

772 The area under the curve for each isotope was extracted using MHQuant software (Agilent). This

773 data was exported as a .csv file and isotopically corrected using an in house modified version of

774 DeuteRater (Naylor et al., 2017)

775

\section{Machine Learning}

777 The selection of the most informative or important features (i.e., the features contributing to the

778 prediction) was performed using a machine-learning approach involving an elastic-net regressor,

779 which followed a round of traditional univariate filtering. The process included two steps:

1. Creating a series of ANOVA models (one for each of the lipid-based features), and preselecting features based on $\eta^{2}$ to limit the complexity of the downstream elastic net model. The uninformative features were rejected and not used in the second step. parameters. The zero coefficients of the model were removed. The top 20 non-zero coefficients provided ranking for the features in terms of their importance.

The elastic net regression attempts to minimize the following functional:

$$
\hat{\beta}=\underset{\beta}{\operatorname{argmin}}\left(\|y-X \beta\|^{2}+\lambda\left((1-\alpha)\|\beta\|^{2} / 2+\alpha|| \beta \|_{1}\right)\right)
$$


respectively. Therefore, the elastic net penalty would become LASSO penalty for $\alpha=1$ and ridge

791 penalty for $\alpha=0$. The parameter $\lambda$ controls the overall strength of the combined penalty term.

792 The input $X$ in the model consists of all the molecular features identified, and y is probability of

793 observing a particular animal.

\section{Statistical Analysis}

796 Statistical analyses of data were performed using one-way ANOVA, a non-parametric Mann-

797 Whitney test, or unpaired Student's t-test depending on the data distribution. $\mathrm{P} \leq 0.05$ were

798 considered statistically significant. Analyses and graphing were performed using Prism

799 (GraphPad v8.0) and R-language for statistical computing.

\section{References}

Amend, S.R., Valkenburg, K.C., and Pienta, K.J. (2016). Murine Hind Limb Long Bone Dissection and Bone Marrow Isolation. J Vis Exp.

806 Anheuser, S., Breiden, B., and Sandhoff, K. (2019). Membrane lipids and their degradation compounds control GM2 catabolism at intralysosomal luminal vesicles. J Lipid Res 60, 1099-

\section{1.}

809 Baars, A., Oosting, A., Lohuis, M., Koehorst, M., El Aidy, S., Hugenholtz, F., Smidt, H., Mischke, M., Boekschoten, M.V., Verkade, H.J., et al. (2018). Sex differences in lipid metabolism are 811 affected by presence of the gut microbiota. Sci Rep 8, 13426.

812 Barron, L., and Wynn, T.A. (2011). Macrophage activation governs schistosomiasis-induced

813 inflammation and fibrosis. European journal of immunology 41, 2509-2514.

814 Beliard, S., Le Goff, W., Saint-Charles, F., Poupel, L., Deswaerte, V., Bouchareychas, L., Huby, T., 815 and Lesnik, P. (2017). Modulation of Gr1(low) monocyte subset impacts insulin sensitivity and 816 weight gain upon high-fat diet in female mice. Int J Obes (Lond) 41, 1805-1814.

817 Berdan, C.A., Erion, K.A., Burritt, N.E., Corkey, B.E., and Deeney, J.T. (2016). Inhibition of 818 Monoacylglycerol Lipase Activity Decreases Glucose-Stimulated Insulin Secretion in INS-1 819 (832/13) Cells and Rat Islets. PloS one 11, e0149008.

820 Boisvert, W.A., Rose, D.M., Johnson, K.A., Fuentes, M.E., Lira, S.A., Curtiss, L.K., and Terkeltaub, 821 R.A. (2006). Up-regulated expression of the CXCR2 ligand KC/GRO-alpha in atherosclerotic 
822 lesions plays a central role in macrophage accumulation and lesion progression. The American

823

824

825

826

827

828

829

830

831

832

833

834

835

836

837

838

839

840

841

842

843

844

845

846

847

848

849

850

851

852

853

854

855

856

857

858

859

860

861

862

863

journal of pathology 168, 1385-1395.

Breese, M.R., and Liu, Y. (2013). NGSUtils: a software suite for analyzing and manipulating nextgeneration sequencing datasets. Bioinformatics 29, 494-496.

Carroll, R.G., Zaslona, Z., Galvan-Pena, S., Koppe, E.L., Sevin, D.C., Angiari, S., Triantafilou, M., Triantafilou, K., Modis, L.K., and O'Neill, L.A. (2018). An unexpected link between fatty acid synthase and cholesterol synthesis in proinflammatory macrophage activation. The Journal of biological chemistry 293, 5509-5521.

Chella Krishnan, K., Mehrabian, M., and Lusis, A.J. (2018). Sex differences in metabolism and cardiometabolic disorders. Curr Opin Lipidol 29, 404-410.

Chen, Y., Lu, J., Huang, Y., Wang, T., Xu, Y., Xu, M., Li, M., Wang, W., Li, D., Bi, Y., et al. (2013).

Association of previous schistosome infection with diabetes and metabolic syndrome: a crosssectional study in rural China. The Journal of clinical endocrinology and metabolism 98, E283287.

Cheng, S.C., Quintin, J., Cramer, R.A., Shepardson, K.M., Saeed, S., Kumar, V., GiamarellosBourboulis, E.J., Martens, J.H., Rao, N.A., Aghajanirefah, A., et al. (2014). mTOR- and HIF-1alphamediated aerobic glycolysis as metabolic basis for trained immunity. Science 345, 1250684. Christ, A., Gunther, P., Lauterbach, M.A.R., Duewell, P., Biswas, D., Pelka, K., Scholz, C.J., Oosting, M., Haendler, K., Bassler, K., et al. (2018). Western Diet Triggers NLRP3-Dependent Innate Immune Reprogramming. Cell 172, 162-175 e114.

Cnop, M., Havel, P.J., Utzschneider, K.M., Carr, D.B., Sinha, M.K., Boyko, E.J., Retzlaff, B.M., Knopp, R.H., Brunzell, J.D., and Kahn, S.E. (2003). Relationship of adiponectin to body fat distribution, insulin sensitivity and plasma lipoproteins: evidence for independent roles of age and sex. Diabetologia 46, 459-469.

Cortes-Selva, D., Elvington, A.F., Ready, A., Rajwa, B., Pearce, E.J., Randolph, G.J., and Fairfax, K.C. (2018). Schistosoma mansoni Infection-Induced Transcriptional Changes in Hepatic Macrophage Metabolism Correlate With an Athero-Protective Phenotype. Front Immunol 9, 2580.

Dobin, A., Davis, C.A., Schlesinger, F., Drenkow, J., Zaleski, C., Jha, S., Batut, P., Chaisson, M., and Gingeras, T.R. (2013). STAR: ultrafast universal RNA-seq aligner. Bioinformatics 29, 15-21. Doenhoff, M.J., Stanley, R.G., Griffiths, K., and Jackson, C.L. (2002). An anti-atherogenic effect of Schistosoma mansoni infections in mice associated with a parasite-induced lowering of blood total cholesterol. Parasitology 125, 415-421.

Douglass, J.D., Zhou, Y.X., Wu, A., Zadroga, J.A., Gajda, A.M., Lackey, A.I., Lang, W., Chevalier, K.M., Sutton, S.W., Zhang, S.P., et al. (2015). Global deletion of MGL in mice delays lipid absorption and alters energy homeostasis and diet-induced obesity. J Lipid Res 56, 1153-1171. Emerging Risk Factors, C., Sarwar, N., Gao, P., Seshasai, S.R., Gobin, R., Kaptoge, S., Di

Angelantonio, E., Ingelsson, E., Lawlor, D.A., Selvin, E., et al. (2010). Diabetes mellitus, fasting blood glucose concentration, and risk of vascular disease: a collaborative meta-analysis of 102 prospective studies. Lancet 375, 2215-2222.

Ewels, P., Magnusson, M., Lundin, S., and Kaller, M. (2016). MultiQC: summarize analysis results for multiple tools and samples in a single report. Bioinformatics 32, 3047-3048. 
864 Fairfax, K.C., Amiel, E., King, I.L., Freitas, T.C., Mohrs, M., and Pearce, E.J. (2012). IL-10R

865

866

867

868

869

870

871

872

873

874

875

876

877

878

879

880

881

882

883

884

885

886

887

888

889

890

891

892

893

894

895

896

897

898

899

900

901

902

903

904

905

906

blockade during chronic schistosomiasis mansoni results in the loss of $B$ cells from the liver and the development of severe pulmonary disease. PLoS pathogens 8, e1002490.

Funk, J.L., Feingold, K.R., Moser, A.H., and Grunfeld, C. (1993). Lipopolysaccharide stimulation of RAW 264.7 macrophages induces lipid accumulation and foam cell formation.

Atherosclerosis 98, 67-82.

Galvan-Pena, S., and O'Neill, L.A. (2014). Metabolic reprograming in macrophage polarization. Front Immunol 5, 420.

Garaude, J., Acin-Perez, R., Martinez-Cano, S., Enamorado, M., Ugolini, M., Nistal-Villan, E., Hervas-Stubbs, S., Pelegrin, P., Sander, L.E., Enriquez, J.A., et al. (2016). Mitochondrial respiratory-chain adaptations in macrophages contribute to antibacterial host defense. Nature immunology 17, 1037-1045.

Girgis, N.M., Gundra, U.M., Ward, L.N., Cabrera, M., Frevert, U., and Loke, P. (2014). Ly6C(high) monocytes become alternatively activated macrophages in schistosome granulomas with help from CD4+ cells. PLoS pathogens 10, e1004080.

Griffin, C., Lanzetta, N., Eter, L., and Singer, K. (2016). Sexually dimorphic myeloid inflammatory and metabolic responses to diet-induced obesity. American journal of physiology. Regulatory, integrative and comparative physiology 311, R211-216.

Hartman, J., and Frishman, W.H. (2014). Inflammation and atherosclerosis: a review of the role of interleukin- 6 in the development of atherosclerosis and the potential for targeted drug therapy. Cardiol Rev 22, 147-151.

Herbert, D.R., Holscher, C., Mohrs, M., Arendse, B., Schwegmann, A., Radwanska, M., Leeto, M., Kirsch, R., Hall, P., Mossmann, H., et al. (2004). Alternative macrophage activation is essential for survival during schistosomiasis and downmodulates $T$ helper 1 responses and immunopathology. Immunity 20, 623-635.

Hinton, W., McGovern, A., Coyle, R., Han, T.S., Sharma, P., Correa, A., Ferreira, F., and de Lusignan, S. (2018). Incidence and prevalence of cardiovascular disease in English primary care: a cross-sectional and follow-up study of the Royal College of General Practitioners (RCGP) Research and Surveillance Centre (RSC). BMJ Open 8, e020282.

Hoerl, A.a.K., RW (1970). Ridge Regression: Biased Estimation for Nonorthogonal Problems. Technometrics 12, 55-67.

Hoogeveen, R.M., Nahrendorf, M., Riksen, N.P., Netea, M.G., de Winther, M.P.J., Lutgens, E., Nordestgaard, B.G., Neidhart, M., Stroes, E.S.G., Catapano, A.L., et al. (2018). Monocyte and haematopoietic progenitor reprogramming as common mechanism underlying chronic inflammatory and cardiovascular diseases. Eur Heart J 39, 3521-3527.

Huang, S.C., Everts, B., Ivanova, Y., O'Sullivan, D., Nascimento, M., Smith, A.M., Beatty, W., Love-Gregory, L., Lam, W.Y., O'Neill, C.M., et al. (2014). Cell-intrinsic lysosomal lipolysis is essential for alternative activation of macrophages. Nature immunology 15, 846-855. Humphries, K.H., Izadnegahdar, M., Sedlak, T., Saw, J., Johnston, N., Schenck-Gustafsson, K., Shah, R.U., Regitz-Zagrosek, V., Grewal, J., Vaccarino, V., et al. (2017). Sex differences in cardiovascular disease - Impact on care and outcomes. Front Neuroendocrinol 46, 46-70. Ifrim, D.C., Quintin, J., Joosten, L.A., Jacobs, C., Jansen, T., Jacobs, L., Gow, N.A., Williams, D.L., van der Meer, J.W., and Netea, M.G. (2014). Trained immunity or tolerance: opposing 
functional programs induced in human monocytes after engagement of various pattern recognition receptors. Clin Vaccine Immunol 21, 534-545. Jung, S.B., Choi, M.J., Ryu, D., Yi, H.S., Lee, S.E., Chang, J.Y., Chung, H.K., Kim, Y.K., Kang, S.G., Lee, J.H., et al. (2018). Reduced oxidative capacity in macrophages results in systemic insulin resistance. Nat Commun 9, 1551.

Kannan, Y., Perez-Lloret, J., Li, Y., Entwistle, L.J., Khoury, H., Papoutsopoulou, S., Mahmood, R., Mansour, N.R., Ching-Cheng Huang, S., Pearce, E.J., et al. (2016). TPL-2 Regulates Macrophage Lipid Metabolism and M2 Differentiation to Control TH2-Mediated Immunopathology. PLoS pathogens 12, e1005783.

Kaufmann, E., Sanz, J., Dunn, J.L., Khan, N., Mendonca, L.E., Pacis, A., Tzelepis, F., Pernet, E., Dumaine, A., Grenier, J.C., et al. (2018). BCG Educates Hematopoietic Stem Cells to Generate Protective Innate Immunity against Tuberculosis. Cell 172, 176-190 e119.

Kim, S.H., and Reaven, G. (2013). Sex differences in insulin resistance and cardiovascular disease risk. J Clin Endocrinol Metab 98, E1716-1721.

Krenkel, O., Hundertmark, J., Abdallah, A.T., Kohlhepp, M., Puengel, T., Roth, T., Branco, D.P.P., Mossanen, J.C., Luedde, T., Trautwein, C., et al. (2020). Myeloid cells in liver and bone marrow acquire a functionally distinct inflammatory phenotype during obesity-related steatohepatitis. Gut 69, 551-563.

Kurylowicz, A., Jonas, M., Lisik, W., Jonas, M., Wicik, Z.A., Wierzbicki, Z., Chmura, A., and Puzianowska-Kuznicka, M. (2015). Obesity is associated with a decrease in expression but not with the hypermethylation of thermogenesis-related genes in adipose tissues. J Transl Med 13, 31.

Lam, R.S., O'Brien-Simpson, N.M., Holden, J.A., Lenzo, J.C., Fong, S.B., and Reynolds, E.C. (2016). Unprimed, M1 and M2 Macrophages Differentially Interact with Porphyromonas gingivalis. PloS one 11, e0158629.

Langston, P.K., Shibata, M., and Horng, T. (2017). Metabolism Supports Macrophage Activation. Front Immunol 8, 61.

Leentjens, J., Bekkering, S., Joosten, L.A.B., Netea, M.G., Burgner, D.P., and Riksen, N.P. (2018). Trained Innate Immunity as a Novel Mechanism Linking Infection and the Development of Atherosclerosis. Circ Res 122, 664-669.

Liang, C.P., Han, S., Senokuchi, T., and Tall, A.R. (2007). The macrophage at the crossroads of insulin resistance and atherosclerosis. Circ Res 100, 1546-1555. Liao, Y., Smyth, G.K., and Shi, W. (2014). featureCounts: an efficient general purpose program for assigning sequence reads to genomic features. Bioinformatics 30, 923-930. Liu, P.S., Wang, H., Li, X., Chao, T., Teav, T., Christen, S., Di Conza, G., Cheng, W.C., Chou, C.H., Vavakova, M., et al. (2017). alpha-ketoglutarate orchestrates macrophage activation through metabolic and epigenetic reprogramming. Nat Immunol 18, 985-994. Luquain-Costaz, C., Lefai, E., Arnal-Levron, M., Markina, D., Sakai, S., Euthine, V., Makino, A., Guichardant, M., Yamashita, S., Kobayashi, T., et al. (2013). Bis(monoacylglycero)phosphate accumulation in macrophages induces intracellular cholesterol redistribution, attenuates liver-X receptor/ATP-Binding cassette transporter A1/ATP-binding cassette transporter G1 pathway, and impairs cholesterol efflux. Arterioscler Thromb Vasc Biol 33, 1803-1811. Marks, J.B., and Raskin, P. (2000). Cardiovascular risk in diabetes: a brief review. J Diabetes Complications 14, 108-115. 
951 Matyash, V., Liebisch, G., Kurzchalia, T.V., Shevchenko, A., and Schwudke, D. (2008). Lipid extraction by methyl-tert-butyl ether for high-throughput lipidomics. J Lipid Res 49, 1137-1146. McCarthy, D.J., Chen, Y., and Smyth, G.K. (2012). Differential expression analysis of multifactor RNA-Seq experiments with respect to biological variation. Nucleic Acids Res 40, 4288-4297. Mehlem, A., Hagberg, C.E., Muhl, L., Eriksson, U., and Falkevall, A. (2013). Imaging of neutral lipids by oil red $O$ for analyzing the metabolic status in health and disease. Nat Protoc 8, 11491154.

958 Moran, A., Jacobs, D.R., Jr., Steinberger, J., Steffen, L.M., Pankow, J.S., Hong, C.P., and Sinaiko, 959 A.R. (2008). Changes in insulin resistance and cardiovascular risk during adolescence:

960 establishment of differential risk in males and females. Circulation 117, 2361-2368.

961 Nascimento, M., Huang, S.C., Smith, A., Everts, B., Lam, W., Bassity, E., Gautier, E.L., Randolph, 962 G.J., and Pearce, E.J. (2014). Ly6Chi monocyte recruitment is responsible for Th2 associated 963 host-protective macrophage accumulation in liver inflammation due to schistosomiasis. PLoS 964 pathogens 10, e1004282. Naylor, B.C., Porter, M.T., Wilson, E., Herring, A., Lofthouse, S., Hannemann, A., Piccolo, S.R., Rockwood, A.L., and Price, J.C. (2017). DeuteRater: a tool for quantifying peptide isotope precision and kinetic proteomics. Bioinformatics 33, 1514-1520. Ng, M.K., Jessup, W., and Celermajer, D.S. (2001). Sex-related differences in the regulation of macrophage cholesterol metabolism. Curr Opin Lipidol 12, 505-510. Nunemaker, C.S., Chung, H.G., Verrilli, G.M., Corbin, K.L., Upadhye, A., and Sharma, P.R. (2014). Increased serum CXCL1 and CXCL5 are linked to obesity, hyperglycemia, and impaired islet function. J Endocrinol 222, 267-276.

973 Oh, D.Y., Morinaga, H., Talukdar, S., Bae, E.J., and Olefsky, J.M. (2012). Increased macrophage migration into adipose tissue in obese mice. Diabetes 61, 346-354. Oiknine, J., and Aviram, M. (1992). Increased susceptibility to activation and increased uptake of low density lipoprotein by cholesterol-loaded macrophages. Arterioscler Thromb 12, 745753.

Opotowsky, A.R., McWilliams, J.M., and Cannon, C.P. (2007). Gender differences in aspirin use among adults with coronary heart disease in the United States. J Gen Intern Med 22, 55-61. Paul, F., Arkin, Y., Giladi, A., Jaitin, D.A., Kenigsberg, E., Keren-Shaul, H., Winter, D., Lara-Astiaso, D., Gury, M., Weiner, A., et al. (2016). Transcriptional Heterogeneity and Lineage Commitment in Myeloid Progenitors. Cell 164, 325. Peters, S.A., Huxley, R.R., and Woodward, M. (2014). Diabetes as risk factor for incident coronary heart disease in women compared with men: a systematic review and meta-analysis of 64 cohorts including 858,507 individuals and 28,203 coronary events. Diabetologia 57, 15421551.

988 Pike Winer, L.S., and Wu, M. (2014). Rapid analysis of glycolytic and oxidative substrate flux of cancer cells in a microplate. PLoS One 9, e109916.

990 Potteaux, S., Gautier, E.L., Hutchison, S.B., van Rooijen, N., Rader, D.J., Thomas, M.J., Sorci-

991 Thomas, M.G., and Randolph, G.J. (2011). Suppressed monocyte recruitment drives macrophage removal from atherosclerotic plaques of Apoe-/- mice during disease regression. The Journal of clinical investigation 121, 2025-2036. 
993 Puleston, D.J., Buck, M.D., Klein Geltink, R.I., Kyle, R.L., Caputa, G., O'Sullivan, D., Cameron, 994 A.M., Castoldi, A., Musa, Y., Kabat, A.M., et al. (2019). Polyamines and elF5A Hypusination

995 Modulate Mitochondrial Respiration and Macrophage Activation. Cell Metab 30, 352-363 e358.

996 Qu, D., Liu, J., Lau, C.W., and Huang, Y. (2014). IL-6 in diabetes and cardiovascular

997 complications. British journal of pharmacology 171, 3595-3603.

998 Robinson, M.D., McCarthy, D.J., and Smyth, G.K. (2010). edgeR: a Bioconductor package for differential expression analysis of digital gene expression data. Bioinformatics 26, 139-140.

1000 Roth, G.A., Johnson, C., Abajobir, A., Abd-Allah, F., Abera, S.F., Abyu, G., Ahmed, M., Aksut, B.,

1001 Alam, T., Alam, K., et al. (2017). Global, Regional, and National Burden of Cardiovascular

1002 Diseases for 10 Causes, 1990 to 2015. J Am Coll Cardiol 70, 1-25.

1003 Rubinow, K.B. (2018). An intracrine view of sex steroids, immunity, and metabolic regulation.

1004 Mol Metab 15, 92-103.

1005 Rudyk, M.P., Pozur, V.V., Voieikova, D.O., Hurmach, Y.V., Khranovska, N.M., Skachkova, O.V.,

1006 Svyatetska, V.M., Fedorchuk, O.G., Skivka, L.M., Berehova, T.V., et al. (2018). Sex-based

1007 differences in phagocyte metabolic profile in rats with monosodium glutamate-induced obesity.

1008 Sci Rep 8, 5419.

1009 Rull, A., Camps, J., Alonso-Villaverde, C., and Joven, J. (2010). Insulin resistance, inflammation,

1010 and obesity: role of monocyte chemoattractant protein-1 (or CCL2) in the regulation of

1011 metabolism. Mediators Inflamm 2010.

1012 Sanya, R.E., Webb, E.L., Zziwa, C., Kizindo, R., Sewankambo, M., Tumusiime, J., Nakazibwe, E.,

1013 Oduru, G., Niwagaba, E., Kabuubi Nakawungu, P., et al. (2019). The effect of helminth infections

1014 and their treatment on metabolic outcomes: results of a cluster-randomised trial. Clinical

1015 infectious diseases : an official publication of the Infectious Diseases Society of America.

1016 Shen, S.W., Lu, Y., Li, F., Shen, Z.H., Xu, M., Yao, W.F., Feng, Y.B., Yun, J.T., Wang, Y.P., Ling, W.,

1017 et al. (2015). The potential long-term effect of previous schistosome infection reduces the risk

1018 of metabolic syndrome among Chinese men. Parasite immunology 37, 333-339.

1019 Shi, L., Pan, H., Liu, Z., Xie, J., and Han, W. (2017). Roles of PFKFB3 in cancer. Signal Transduct

1020 Target Ther 2, 17044.

1021 Stanley, R.G., Jackson, C.L., Griffiths, K., and Doenhoff, M.J. (2009). Effects of Schistosoma

1022 mansoni worms and eggs on circulating cholesterol and liver lipids in mice. Atherosclerosis 207,

1023 131-138.

1024 Stienstra, R., Netea-Maier, R.T., Riksen, N.P., Joosten, L.A.B., and Netea, M.G. (2017). Specific

1025 and Complex Reprogramming of Cellular Metabolism in Myeloid Cells during Innate Immune

1026 Responses. Cell Metab 26, 142-156.

1027 Tacke, F., Alvarez, D., Kaplan, T.J., Jakubzick, C., Spanbroek, R., Llodra, J., Garin, A., Liu, J., Mack, 1028 M., van Rooijen, N., et al. (2007). Monocyte subsets differentially employ CCR2, CCR5, and

1029 CX3CR1 to accumulate within atherosclerotic plaques. The Journal of clinical investigation 117,

$1030 \quad 185-194$.

1031 Tan, Y.Y., Gast, G.C., and van der Schouw, Y.T. (2010). Gender differences in risk factors for

1032 coronary heart disease. Maturitas 65, 149-160.

1033 Taneja, V. (2018). Sex Hormones Determine Immune Response. Front Immunol 9, 1931.

1034 Tibshirani, R. (1996). Regression shrinkage and selection via the lasso. J. R. Statist. Soc. B 58,

1035 267-288. 
1036 van der Windt, G.J., Everts, B., Chang, C.H., Curtis, J.D., Freitas, T.C., Amiel, E., Pearce, E.J., and 1037 Pearce, E.L. (2012). Mitochondrial respiratory capacity is a critical regulator of CD8+ T cell

1038 memory development. Immunity 36, 68-78.

1039 Vats, D., Mukundan, L., Odegaard, J.I., Zhang, L., Smith, K.L., Morel, C.R., Wagner, R.A., Greaves, 1040 D.R., Murray, P.J., and Chawla, A. (2006). Oxidative metabolism and PGC-1beta attenuate 1041 macrophage-mediated inflammation. Cell Metab 4, 13-24.

1042 Vernia, S., Cavanagh-Kyros, J., Barrett, T., Jung, D.Y., Kim, J.K., and Davis, R.J. (2013). Diet-

1043 induced obesity mediated by the JNK/DIO2 signal transduction pathway. Genes Dev 27, 2345-

10442355.

1045 Willeit, J., Kiechl, S., Egger, G., Oberhollenzer, M., Oberhollenzer, F., Muggeo, M., Poewe, W., 1046 and Bonora, E. (1997). The role of insulin in age-related sex differences of cardiovascular risk 1047 profile and morbidity. Atherosclerosis 130, 183-189.

1048 Winn, N.C., Jurrissen, T.J., Grunewald, Z.I., Cunningham, R.P., Woodford, M.L., Kanaley, J.A., 1049 Lubahn, D.B., Manrique-Acevedo, C., Rector, R.S., Vieira-Potter, V.J., et al. (2019). Estrogen 1050 receptor-alpha signaling maintains immunometabolic function in males and is obligatory for 1051 exercise-induced amelioration of nonalcoholic fatty liver. Am J Physiol Endocrinol Metab 316, 1052 E156-E167.

1053 Wiria, A.E., Hamid, F., Wammes, L.J., Prasetyani, M.A., Dekkers, O.M., May, L., Kaisar, M.M., 1054 Verweij, J.J., Guigas, B., Partono, F., et al. (2015). Infection with Soil-Transmitted Helminths Is 1055 Associated with Increased Insulin Sensitivity. PloS one 10, e0127746.

1056 Wolde, M., Berhe, N., Medhin, G., Chala, F., van Die, I., and Tsegaye, A. (2019). Inverse 1057 Associations of Schistosoma mansoni Infection and Metabolic Syndromes in Humans: A Cross1058 Sectional Study in Northeast Ethiopia. Microbiol Insights 12, 1178636119849934.

1059 Wolfs, I.M., Stoger, J.L., Goossens, P., Pottgens, C., Gijbels, M.J., Wijnands, E., van der Vorst, 1060 E.P., van Gorp, P., Beckers, L., Engel, D., et al. (2014). Reprogramming macrophages to an anti1061 inflammatory phenotype by helminth antigens reduces murine atherosclerosis. FASEB J 28, 2881062299.

1063 Wong, N.D., Zhao, Y., Patel, R., Patao, C., Malik, S., Bertoni, A.G., Correa, A., Folsom, A.R., 1064 Kachroo, S., Mukherjee, J., et al. (2016). Cardiovascular Risk Factor Targets and Cardiovascular 1065 Disease Event Risk in Diabetes: A Pooling Project of the Atherosclerosis Risk in Communities 1066 Study, Multi-Ethnic Study of Atherosclerosis, and Jackson Heart Study. Diabetes Care 39, 6681067676.

1068 Zhou, Z., Subramanian, P., Sevilmis, G., Globke, B., Soehnlein, O., Karshovska, E., Megens, R., 1069 Heyll, K., Chun, J., Saulnier-Blache, J.S., et al. (2011). Lipoprotein-derived lysophosphatidic acid 1070 promotes atherosclerosis by releasing CXCL1 from the endothelium. Cell Metab 13, 592-600. 1071 Zou, H.a.H., T (2005). Regularization and variable selection via the elastic net. Journal of the 1072 Royal Statistical Society 67, 301-320.

1073

1074

1075

Figure legends

1076 Figure 1. Bone marrow derived macrophages (BMDM) from ApoE ${ }^{-/-}$male S. mansoni

1077 infected mice exhibit increased oxygen consumption and mitochondria mass. ApoE ${ }^{-/-}$male 
1078 were fed HFD for 10 days before infection with $S$. mansoni. Ten weeks post infection mice were

1079 sacrificed and bone marrow cells were harvested and cultured for 7 days under M-CSF. (A)

1080 SeaHorse assay results for OCR of BMDC from infected and uninfected $\mathrm{ApoE}^{-/-}$males in basal

1081 conditions and in response to mitochondrial inhibitors. (B) Quantification (in picomoles per

1082 minute) of the basal oxygen consumption of BMDM from uninfected or infected $\mathrm{ApoE}^{-/-} \mathrm{HFD}$

1083 male mice. (C) Quantification of the spare respiratory capacity of BMDM from uninfected or

1084 infected ApoE - $^{-/}$HFD male mice (D) Extracellular acidification rate of BMDM from male

1085 uninfected or infected $\mathrm{ApoE}^{-/}$. (E) Oil Red O relative staining in BMDM from ApoE ${ }^{-/-}$mice $(\mathrm{F})$

1086 MitoTracker Red Deep Stain measure by flow cytometry in BMDM from ApoE ${ }^{-/-}$mice.

1087 SeaHorse assay analysis were performed the Seahorse XFe96 instrument. ${ }^{*} \mathrm{p}<0.05 ; * *^{*}<0.01$;

$1088 * * * \mathrm{p}<0.001$. Graphs are representative of multiple experiments $(2-3)$, with $\mathrm{n}>4$ per group.

1090 Figure 2.

1091 BMDM from male $S$. mansoni infected HFD $\mathbf{A p o E}^{-/-}$mice have increased TCA cycle usage

1092 and significantly reduced cholesterol esters. A-E) M $\phi$ were differentiated from bone marrow

1093 of 10-week infected animals with M-CSF in a 7-day culture in normal glucose and then switched

1094 to ${ }^{13} \mathrm{C}$-labeled glucose for 24 hours. F-H) M $\phi$ s were differentiated with M-CSF in a 7-day culture

1095 and then total cellular lipids were extracted and analyzed via LC-MS based lipidomic analysis.

1096 F) PLS-DA derived score from HFD Infected and HFD Uninfected BMDM. G) Plot of lipid

1097 species from the BMDM of infected and uninfected males on HFD, significantly altered lipid

1098 species are red and labeled. H)Table of statistical analysis of VIP I) Box whisker plot of

1099 normalized AUC of cholesterol ester species, which were identified as VIP compounds from the

1100 PLS-DA analysis. J-L) Bone marrow macrophages were differentiated with M-CSF and mRNA

1101 sequenced via RNASeq. J) Volcano plot of significantly differentially expressed genes between 
1102 BMDM from S. mansoni infected uninfected mice. K) iPathway analysis showed distinct profiles

1103 in BMDM from S. mansoni infected, mice. L,M) Total RNA was extracted from biologically

1104 independent BMDM differentiated in M-CSF for Real-time PCR validation of mgll and slc1a3

1105 regulation.

1106 Data in A-I are representative of 3 experiments with 4-6 mice per group in each experiment.

1107 Data in $\mathrm{J}$ and $\mathrm{K}$ are representative of sequencing of 2 biologically independent experiments with

1108 5-6 mice per group. Data in L are from 2 biologically independent experiments with 4-6 mice per

1109 group.

1111 Figure 3

1112 S. mansoni infection does not protect females from metabolic disease despite inducing

1113 alternative activation in hepatic macrophages. (A-B) Total body weight and glucose tolerance

1114 test (GTT) at 10 weeks post-infection. GTT values were analyzed by Area Under the Curve and

1115 graphed using GraphPad Prims. (C) (D) Flow cytometry analysis of alternative activation

1116 markers CD206 and CD302 in perfused and digested livers gated on hepatic macrophages

$1117\left(\mathrm{CD} 5^{+} \mathrm{CD} 64^{+} \mathrm{MertK}^{+}\right)$Arg-1 expression in hepatic macrophages from uninfected and infected

$1118 \mathrm{ApoE}^{-/-}$mice Nos-2 expression by flow cytometry in hepatic macrophages 10 weeks post

1119 infection. (E) Ly6C expression in monocyte from peripheral blood mononuclear cells (PBMC) at

1120 10-week post infection by flow cytometry (F) MitoTracker Red in CD115 monocytes from

1121 PBMC at 10 weeks post immunization in uninfected and infected $\mathrm{ApoE}^{-/-}$mice. (G) Total cell

1122 counts from bone marrow cells utilizing trypan-blue discrimination of apoptotic cells $(\mathrm{H})$

1123 Percentages of CMP (Lin $\left.{ }^{-C D} 127^{-} \mathrm{c}-\mathrm{Kit}^{+} \mathrm{Sca}-1^{-} \mathrm{CD} 34^{+} \mathrm{CD} 16 / 32^{\text {low }}\right)$ and GMP (Lin ${ }^{-} \mathrm{CD} 12^{-} \mathrm{c}-$

$\left.1124 \mathrm{Kit}^{+} \mathrm{Sca}-1^{-\mathrm{CD}} 34^{+} \mathrm{CD} 16 / 32^{\mathrm{hi}}\right)(\mathrm{I}) \mathrm{CMP}$ cell counts in bone marrow $\mathrm{ApoE}^{-/-}(\mathrm{J}) \mathrm{GMP}$ cell counts 10 
1125 week post infection in ApoE ${ }^{-/-}$mice (K) Flow cytometry analysis of MDP defined as Lin`c-

$1126 \mathrm{Kit}^{+} \mathrm{Sca}-1^{-} \mathrm{Fc} \gamma \mathrm{R}^{\text {low }} \mathrm{CD} 34^{+} \mathrm{CD} 115^{+}(\mathrm{L}) \mathrm{MDP}$ cell counts in ApoE ${ }^{-/}$uninfected and infected male

1127 mice. Graphs are representative from experiments that were performed 3-4 times with $n>4$.

1128 Exception is MitoTracker data, which was performed twice, with $n>4$. Statistical analysis was

1129 done using unpaired Student's t test, *p $<0.05 ; *$; $<0.01$.

1131 Figure 4.

1132 S. mansoni infection induces sex-specific modulation of oxygen consumption and beta-

1133 oxidation in BMDM. (A-C) oxygen consumption rate and spare respiratory capacity were

1134 measured at steady state. D-F) BMDM palmitate dependent oxygen consumption and spare

1135 respiratory capacity was measured in glucose limiting conditions. G) Oil Red O relative staining

1136 in BMDM from ApoE ${ }^{-/-}$mice $(\mathrm{H})$ MitoTracker Red Deep Stain measure by flow cytometry in

1137 BMDM from ApoE $\mathrm{E}^{-/}$mice. SeaHorse assay analysis were performed the Seahorse XFe96

1138 instrument. ${ }^{*} \mathrm{p}<0.05 ; * * \mathrm{p}<0.01 ; * * \mathrm{p}<0.001$. Graphs are representative of multiple

1139 experiments (2-3), with $\mathrm{n}>4$ per group.

1141 Figure 5.

1142 S. mansoni infection induces significantly different cellular lipid profiles in BMDM from

1143 HFD female and male ApoE ${ }^{-/-}$mice. A) PLS-DA derived score of LC-MS based lipidomic

1144 analysis of BMDM from HFD Infected females vs males. B) Box whisker plot of normalized

1145 AUC of cholesterol ester species, between female and male infected and uninfected HFD ApoE ${ }^{-/-}$

1146 mice. C) PLS-DA derived score from Female HFD Infected and uninfected BMDM. D) Dot plot

1147 of lipid species identified in BMDM from infected and uninfected female $\mathrm{ApoE}^{-/-}$mice on HFD, 
1148 significant lipid species are represented by red dots and labeled. E) Relative quantitation of BMP

1149 compounds in BMDM from male and female animals with and without S. mansoni infection. F-I)

1150 Macrophages were differentiated with M-CSF in a 7-day culture with normal glucose and serum,

1151 and then switched to ${ }^{13} \mathrm{C}$-labeled palmitate in dialyzed serum for 36 hours. J-N) Culture

1152 supernatants from BMDM stimulated with LPS for 24 hours were assayed for

1153 chemokines/cytokines. Data in A-E and J-N are representative of 2 biologically independent

1154 experiments with 4-8 mice per group. F-I are one experiment with 5-6 mice per group.

1158 Figure 6.

1159 S. mansoni infection differentially regulates the transcriptomes of BMDM from female and

1160 male $\mathbf{A p o E}^{-/-}$mice. (A) Venn diagram showing upregulated genes in males only, independently

1161 of infection state and pathway analysis corresponding to the identified genes. (B) Venn diagram

1162 and pathway analysis of differentially expressed genes in males compared to females. (C) Gene

1163 ontology map of differentially expressed genes in response to $S$. mansoni infection in male

1164 compared to female ApoE ${ }^{-/}$mice on HFD. Sequencing data are from one experiment with 5-6

1165 mice per group.

1167 Figure 7. S. mansoni induced modulation of male macrophage metabolism is long-lived in

1168 the absence of antigen. Bone marrow from 10-week S. mansoni infected or control ApoE-/-

1169 mice on HFD was transferred into busulfan treated ApoE ${ }^{-/-}$recipients on HFD. A,B) Glucose

1170 tolerance test (GTT) at 10 weeks post-infection. GTT values were analyzed by Area Under the

1171 Curve and graphed using GraphPad Prism. C-E) Oxygen consumption rate and spare respiratory 
bioRxiv preprint doi: https://doi.org/10.1101/2020.04.20.050898; this version posted May 21, 2020. The copyright holder for this preprint (which

was not certified by peer review) is the author/funder, who has granted bioRxiv a license to display the preprint in perpetuity. It is made available under aCC-BY 4.0 International license.

1172 capacity were measured at steady state in 7day BMDM. F) MitoTracker Red Deep Stain measure

1173 by flow cytometry in BMDM. Data is two combined experiments 7-8 animals per group.

1174 Statistical analysis was done using Welch's t-tests.

1175 
Figure 1

A B

C

D

E

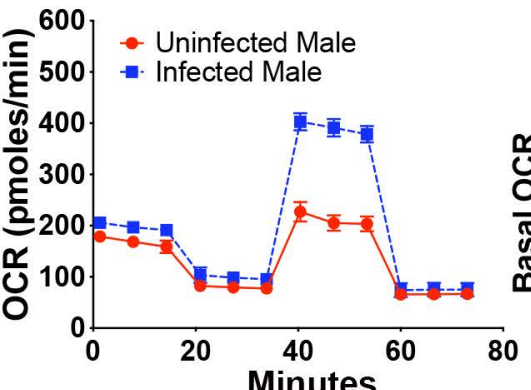

Minutes
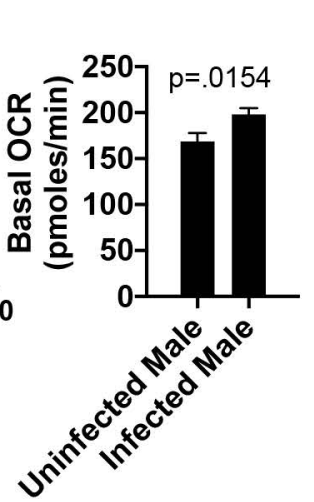
र०

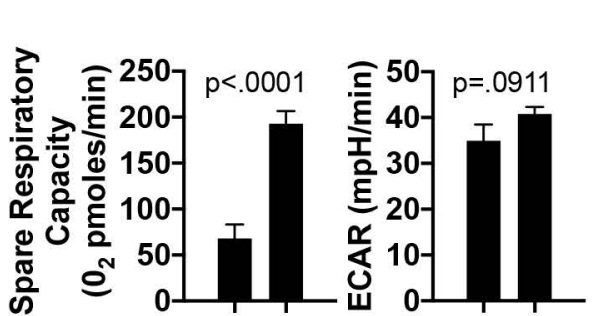
1e
5"

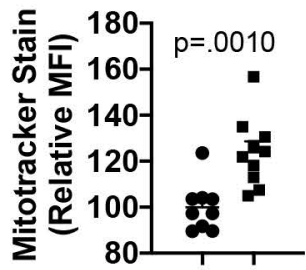

ō

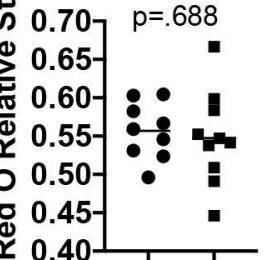
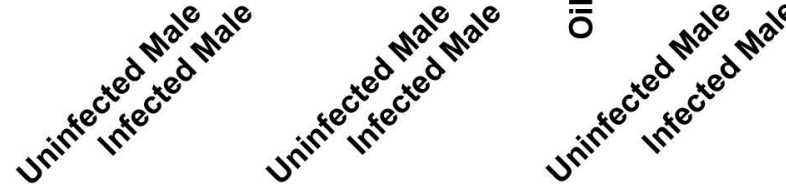
Figure 2
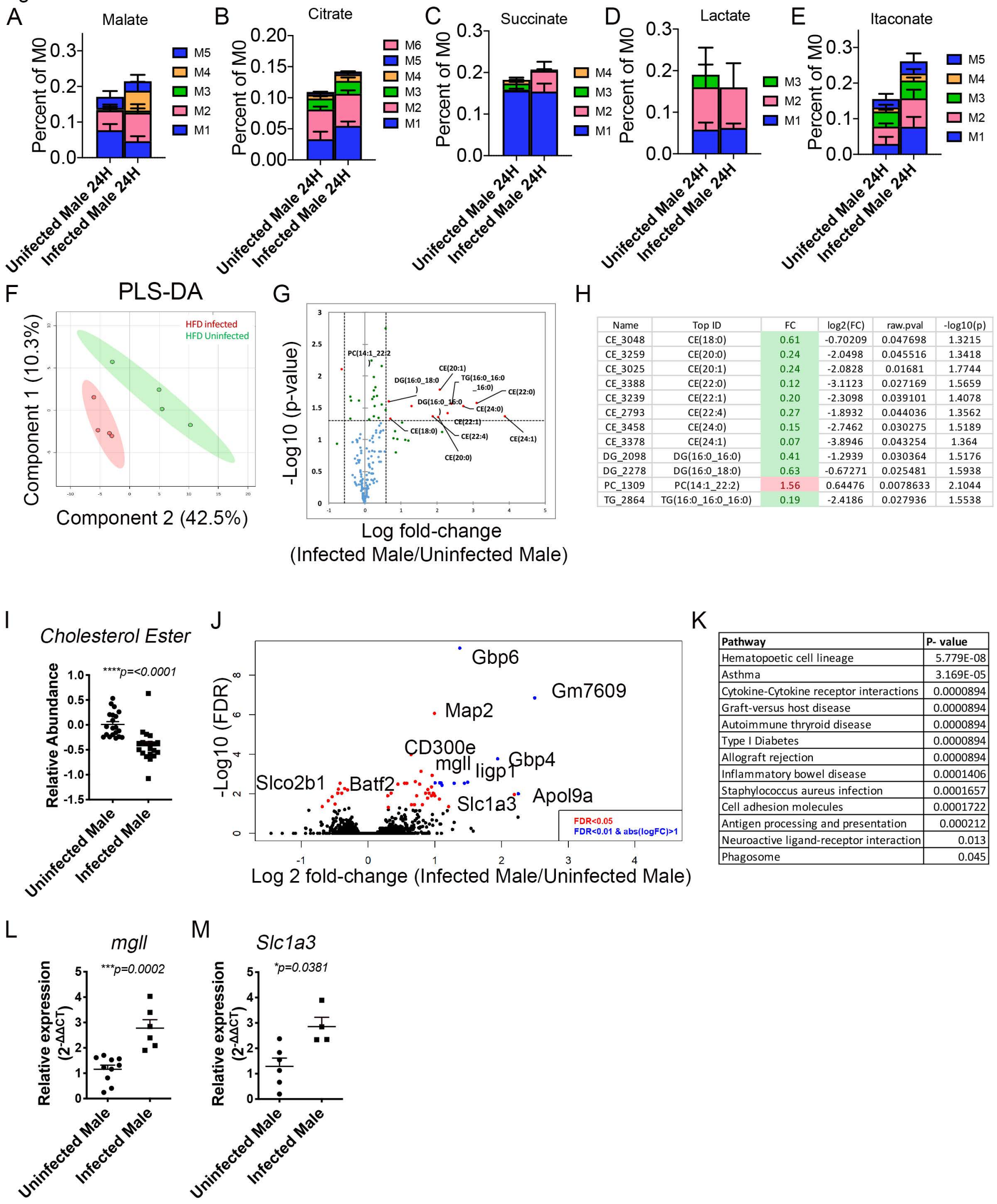
A Female
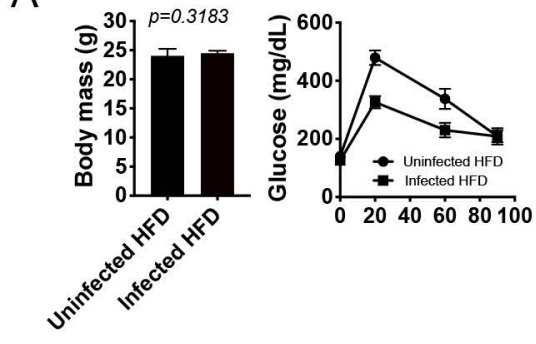

D

Liver

Naive

CD45+CD64 ${ }^{+}$MertK ${ }^{+}$

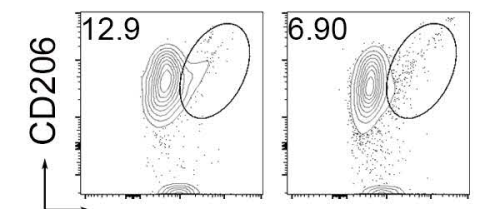

CD301

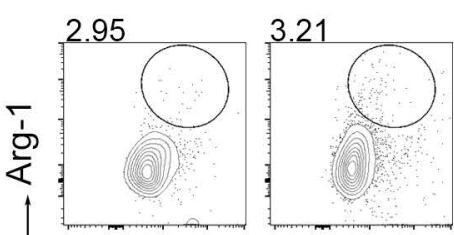

CD301

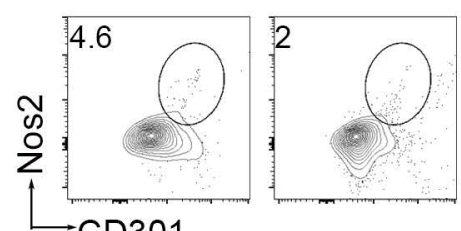

CD301

Female Male

$\mathrm{H}$

Naive

Female Male

10 Week S. mansoni

Lin-CD127-C-Kit+Sca-1-CD34+

ํํำ

21.674 .8

CD16/32

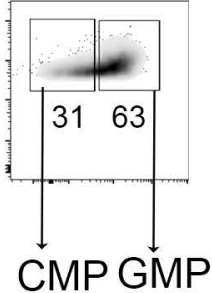

Naive

ד্ঠ

ז্்

Linc-Kit'Sca-1-FcyR RowCD34

10 Week S. mansoni
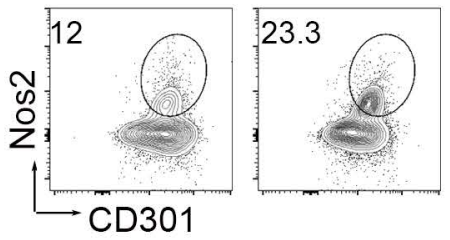

Female Male
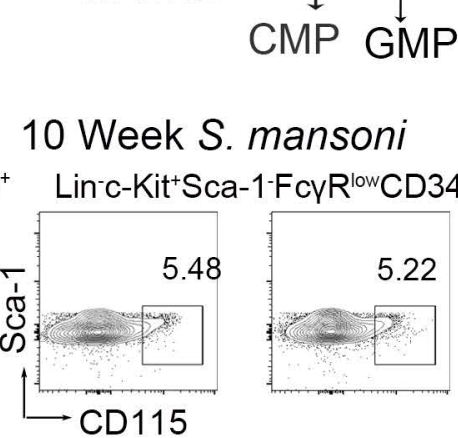

E

\section{PBMC}

Naive

Monocytes
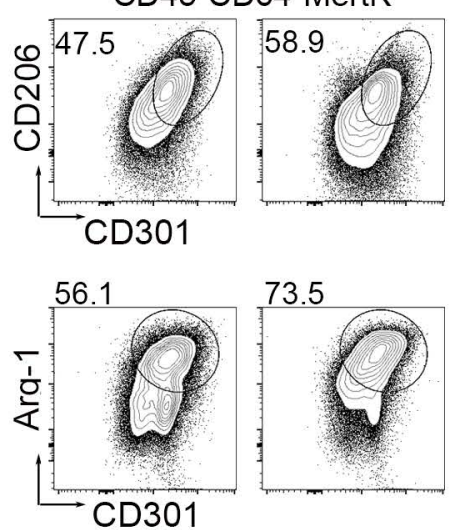

$73.5 \quad \mathrm{~F}$

F

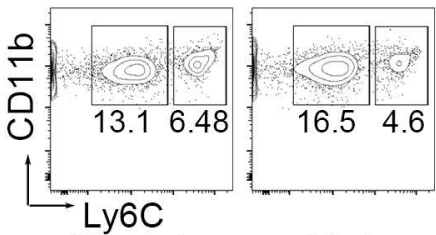

Ly6e

ல̀

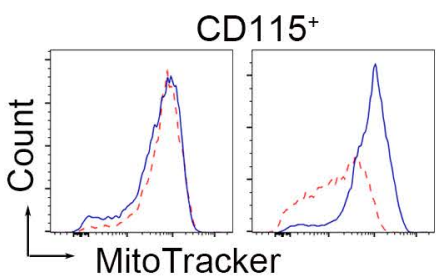

Female Male

Naive

-10 Week S. mansoni
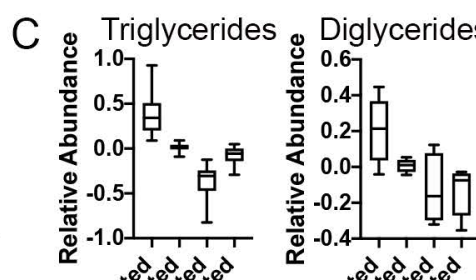

0

बिए

10 Week S. mansoni Monocytes

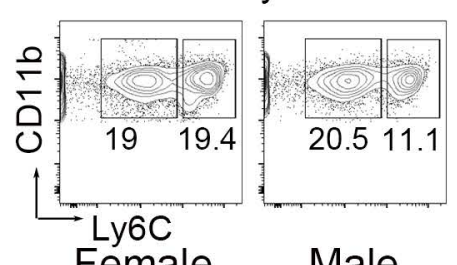

$G$

Female

Male

Total Bone Marrow

Female
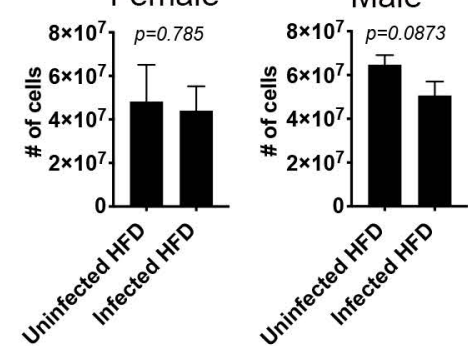

I

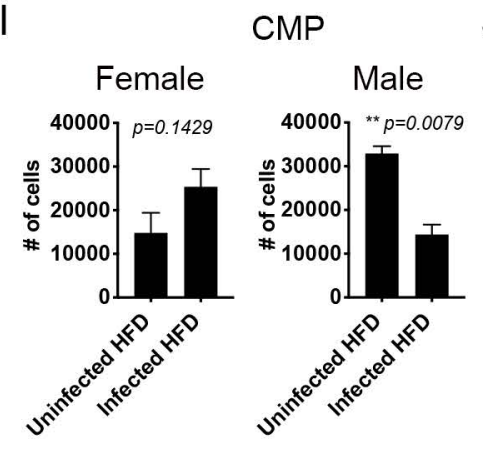

L

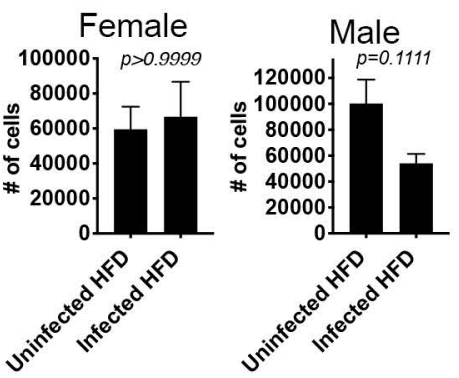

JMP

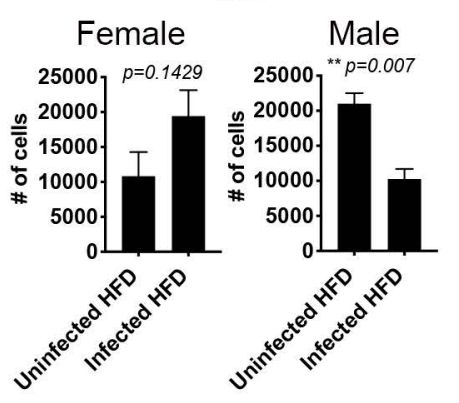


Figure 4
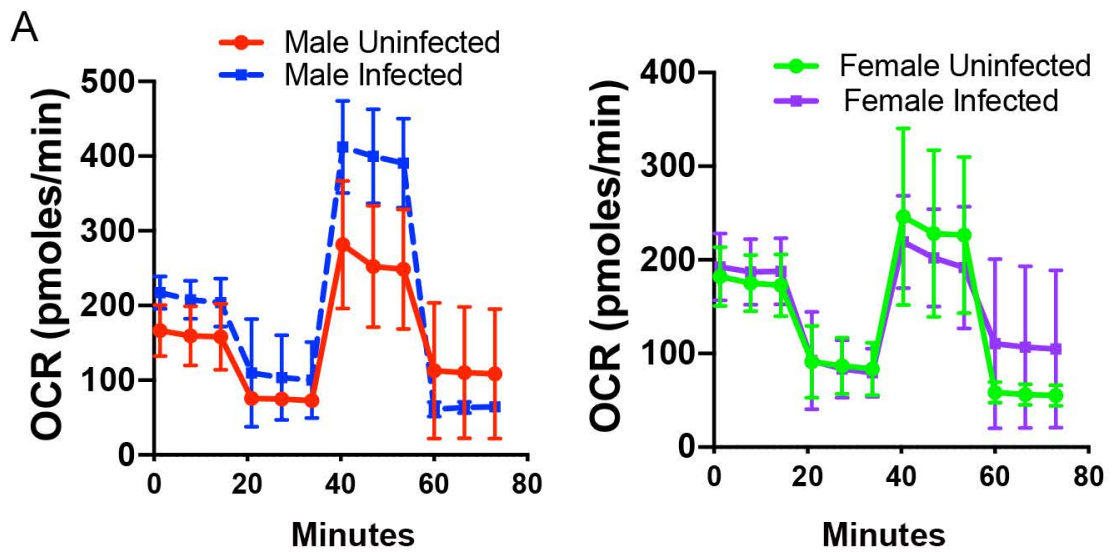

B
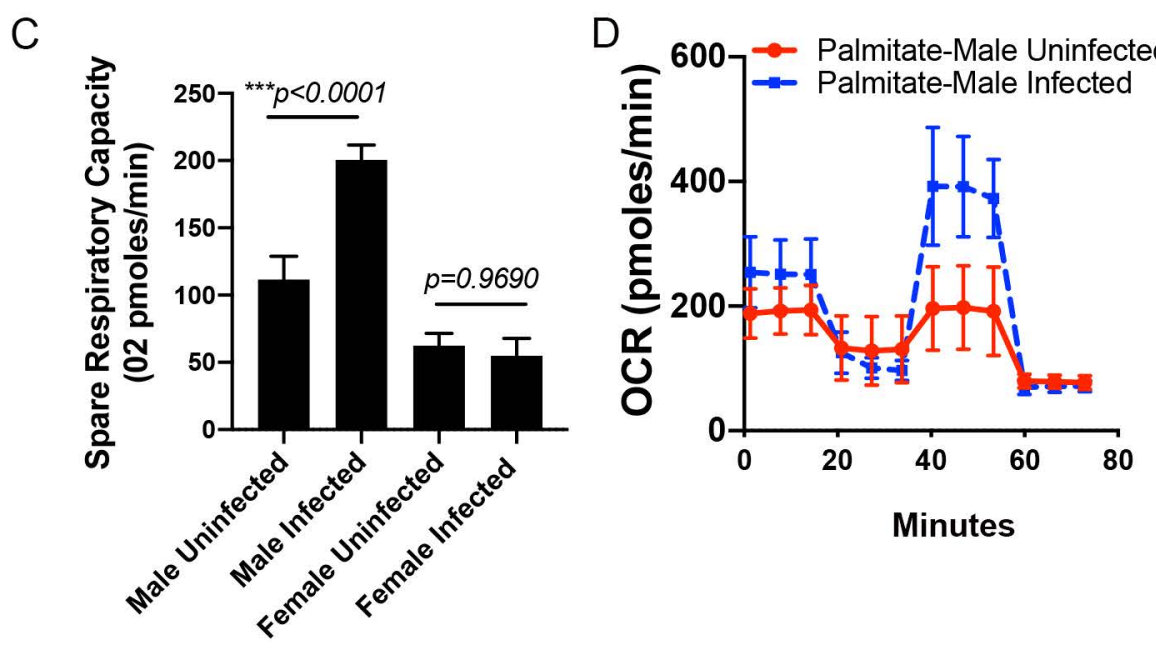

E

F

G
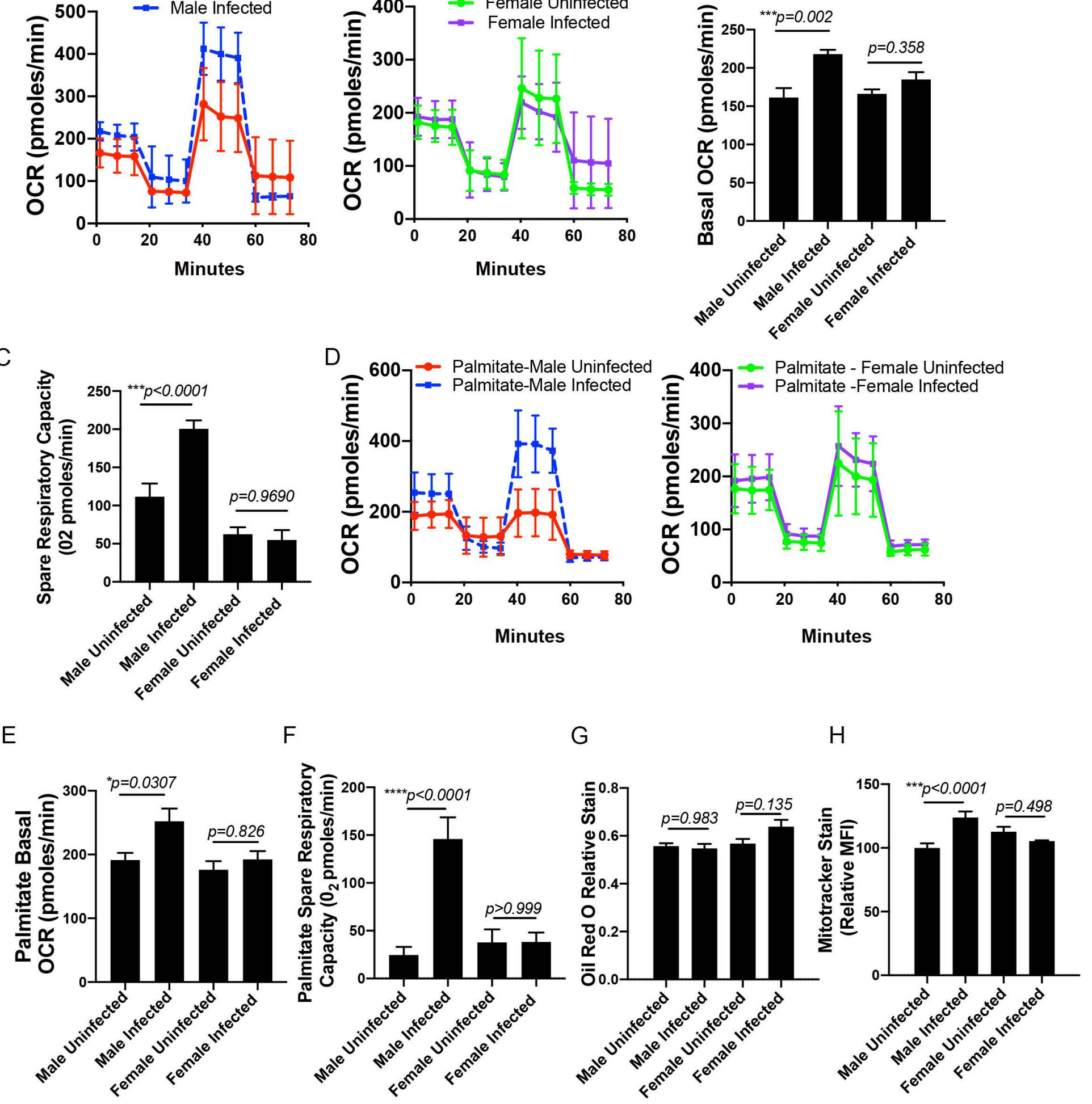
